\title{
11. Über die Überlagerungsgruppe und die stetige projektive Darstellung topologischer Gruppen.
}

\author{
Von Yukiyosi KaWADA.
}

(Eingegangen am 13. Mai, 1940.)

Die vorliegende Arbeit besteht aus zwei Teilen.

Der erste Teil behandelt die Theorie der Überlagerungsgruppe topologischer Gruppen. Die Theorie der Überlagerungsgruppe ist für die zusammenhängende, im Kleinen zusammenhängende und im Kleinen einfach-zusammenhängende Gruppe von $\mathrm{O}$. Schreier in seinen bekannten Arbeiten $\left({ }^{1}\right)$ ausgiebig entwickelt worden. Wir wollen eine entsprechende Theorie für diejenige Gruppe begründen, die zusammenhänged, im Kleinen zusammenhängend aber nicht notwendig im Kleinen einfach-zusammenhängend ist. Unser Ansatz zur Definition der Überlagerungsgruppe ist von L. Vietoris entnommen $\left({ }^{2}\right)$.

Dieser Teil würde also, wie uns scheint, vom selbständigen Interesse sein; gilt jedoch als Vorbereitung zum nachfolgenden zweiten Teil.

Der zweite Teil behandelt die Theorie der stetigen projektiven Darstellung (S.P.D.) topologischer Gruppen. Das bekannteste Beispiel solcher Darstellung ist die ,, Spindarstellung " orthogonaler Gruppen, die von vielen Autoren recht eingehend untersucht worden ist $\left({ }^{3}\right)$. Hier soll eine allgemeine Therie der S.P.D. begründet werden $\left({ }^{4}\right)$.

(1) O. Schreiger, Abstrakte kontinuierliche Gruppen, Abh. Math. Sem. Hamburg, 4 (1926), $15 \cdot 32$.

O. Schreier, Verwandtschaft stetiger Gruppen im Grossen, Abh. Math. Sem. Hamburg, 5 (1926), 233-244; zitiert mit S. 1. und S. 2. Vgl. auch L. Pontrjagin, Topological groups, (1939).

(2) L. Vietoris, Über den höheren Zusammenhang kompakter Räume, Math. Ann., 97 (1928), S. 459.

Vgl. auch W. Hurewicz, Beiträge zur Theorie der Deformationen, IV. Asphärische Räume, Proc. Royal Acad. Amsterdam, 39 (1936), 215-224.

H. Freudenthal, Entwicklungen von Räumen und ihre Gruppen, Composition Math., 4 (1937), 145-234.

(3) Vgl. z.B. H. Weyl, The classical groups, (1939), S. 270.

(4) Vgl. H. Weyl, Gruppentheorie und Quantenmechanik, (1928), S. 147. Bzgl. der allgemeinen Theorie der projektiven Darstellung der Gruppen sieh auch M. Tazawa, Über die Darstellung der beliebigen Gruppen durch gebrochene lineare Transformationen, Sci. Rep. Tohoku Univ., I. Series, 23 (1934), 352-371. 
Mittels derjenigen Idee, die von I. Schur bei der Untersuchung der projektiven Darstellung endlicher Gruppen verwendet wurde $\left({ }^{5}\right)$, werden wir einen Sats (Satz 11) erhalten, der uns gestattet, die Untersuchung der S.P.D. einer topologischer Gruppe (5) auf die der stetigen ganzen Darstellung einer endlichvielblättrigen Ủberlagerungsgruppe von (\$) zu reduzieren. Nach diesem Satz wird ferner der ,, Multiplikator", d.h. die Gruppe der Faktorensysteme in engem Zusammenhang mit der Fundamentalgruppe gebrachtet (Satz 15, 15'). Über den Multiplikator der kompakten, metrischen, zusammenhängenden im kleimen zusammenhänaenden Gruppe gelten viel einfachere Sätze als bei diskreten (Satz 16, 17).

\section{$\S 1$. Überlagerungsgruppe.}

1. Es sei (3) eine topologische Gruppe und $u$ eine Umgebung von dem Einselement $e$ von (9), die der Bedingung $\mathfrak{u}=\mathfrak{u}^{-1}$ genügt. Unter einem $\mathfrak{u}-W e g W(a, b)$ für $\mathfrak{i}$ verstehen wir eine geordnete Menge endlich vieler Elemente aus (5)

$$
\left\{a, c_{1}, \ldots, c_{r}, b\right\} \text {, }
$$

sodass $a^{-1} c_{1}, c_{r}^{-1} b$ und $c_{n}^{-1} c_{n+1}$ alle zu $u$ gehören $(n=1, \ldots, r)$. Wir nennen dabel $a, b$ das Anfangselement bzw. das Endelement von $W$, und sagen, dass a mit $b$ veroindet.

Eine Teilmenge $\mathfrak{M}$ von $(\mathfrak{S})$ (die such $=(\mathfrak{3}$ sein kann) heisst $\mathfrak{H}$-zusammenhängend (u-z.h.), wenn jedes Elementenpaar aus $\mathfrak{M}$ durch einen ganz auf $\mathfrak{M}$ liegenden $\mathfrak{u}-\mathrm{Weg}$, d.h. durch einen $\mathfrak{u}-\mathrm{Weg}$, dessen sämtlichen Elemente zu $\mathfrak{M}$ gehören, verbunden wird; und $\mathrm{O}$-zusammenhängend (O-z.h.), wenn $\mathfrak{M}$ für jede Umgebung $\mathfrak{u}$ von $e$ mit $\mathfrak{u}=\mathfrak{u}^{-1} \mathfrak{u}$-z.h. ist. Die im üblichen Sinne zusammenhängende Gruppe ist O-z.h.. und umgekehrt ist jede kompakte metrische O-z.h. Gruppe z.h. $\left({ }^{6}\right)$

Wir nennen (S) im Kleinen O-z.h., falls jede Umgebung von $e$ eine O-z.h. Umgebung enthält. Eine im Kleinen zusammenhängende Gruppe ist im Kleinen O-z.h.; umgekehrt gilt, wie man leicht einsehen kann, dass, bei jeder vollständigen, O-zusammenhüngenden und im Kleinen $\mathrm{O}$ -

(5) I. Schur, Über die Darstellung der endlichen Gruppen durch gebrcchene lineare Substitutionen, Crelles Journal, 127 (1904), 2050.

K. Asano und K. Shoda, Zur Theorie der Darstellung einer endlichen Gruppe durch Kollineationen, Compositio Math., 2 (1935), 230-240.

Für die weiteren Literaturen vgl. B. L. van der Waerden, Gruppen von linearen Transformationen, Ergebnisse d. Math. (1935), § 21.

(6) Vgl. P. Alexandroff und H. Hopf, Topologie, S. 117, Satz 1. 
zusammenhängenden Gruppe, jedes Element $a \in(5)$ mit $e$ durch einen stetigen Weg verbindbar ist, und dass, bei jeder solchen Gruppe jede Umgebung $\mathfrak{u}$ von $e$ eine solche Umgebung $\mathfrak{b}$ enthält, dass jedes Element aus $\mathfrak{b}$ durch einen stetigen Weg in $u$ mit $e$ verbunden wird.

Von nun an wollen wir zwei tt-Wege:

$$
\begin{aligned}
& \left\{a_{1}, a_{2}, \ldots, a_{n-1}, a_{n}, a_{n+1}, \ldots, a_{r}\right\} \text { und } \\
& \left\{a_{1}, a_{2}, \ldots, a_{n-1}, a_{n+1}, \ldots, a_{r}\right\}
\end{aligned}
$$

als identisch betrachten, wenn $a_{n}=a_{n-1}$ oder $a_{n}=a_{n+1}$ ist. Zwei 1i-Wege

$$
W(p, q)=\left\{p, a_{1}, \ldots, a_{r}, q\right\} \text { und } W^{\prime}(p, q)=\left\{p, a_{1}^{\prime}, \ldots, a_{r}^{\prime}, q\right\}
$$

sollen $\mathfrak{u}$-benachbart heissen, in Zeichen:

$$
W(p, q) \approx W^{\prime}(p, q) \quad(\mathfrak{t}),
$$

falls $a_{n}^{-1} a_{n}^{\prime} \in \mathfrak{t t}(n=1, \ldots, r)$ (oder $a_{n}^{\prime-1} a_{n} \in \mathfrak{u}^{-1}=\mathfrak{t}$ ) ist. $W(p, q)$ und $W(p, q)$ heissen $\mathfrak{u}$-homotop, in Zeichen :

$$
W(p, q) \sim W^{\prime}(p, q) \quad(\mathfrak{u}),
$$

wenn man endlich viele $\mathfrak{u}$-Wege $W_{n}(p, q)$ derart finden kann, dass

$$
W(p, q) \approx W_{1}(p, q) \approx W_{2}(p, q) \approx \cdots \approx W_{r}(p, q) \approx W^{\prime}(p, q)(\mathfrak{t}) .
$$

gilt. Ein geschlossener $\mathfrak{u}$-Weg $W(e, e)$, der mit $\{e, e\} \mathfrak{u}$-homotop ist heisse $\mathfrak{u}$-homotop $O$.

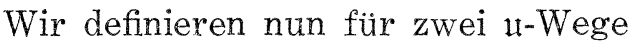

$$
W(e, a)=\left\{e, a_{1}, \ldots, a_{r}, a\right\} \text { und } W(e, b)=\left\{e, b_{1}, \ldots, b_{s}, b\right\}
$$

mit dem Anfangselement $e$ den Produktweg $W(e, a) \cdot W(e, b)=W(e, a b)$ durch

$$
W(e, a b)=\left\{e, a_{1}, \ldots, a_{r}, a, a b_{1}, a b_{2}, \ldots, a b_{r}, a b\right\} .
$$

Dann bildet die Gesamtheit aller 1t-Wege mit dem Anfangselement $e$ bei dieser Multiplikationsvorschrift eine Gruppe $\mathfrak{S}_{0}(u)$; der inverse Weg von $W(e, a)$ ist dabei

$$
W\left(e, a^{-1}\right)=\left\{e, a^{-1} a_{r}, \ldots, a^{-1} a_{1}, a^{-1}\right\},
$$

und die Eins $\{e, e\}$. Die Gesamtheit aller geschlossenen $\mathfrak{u}$-Wege, die $\mathfrak{u}$-homotop $O$ sind, bildet einen Normalteiler $\Re^{0}$ von $2 \mathfrak{S}_{\mathfrak{G}}^{0}(\mathfrak{u})$; die Faktorgruppe $\mathfrak{W}_{\mathscr{S}}^{0}(\mathfrak{u}) / \mathfrak{R}^{0}$ nennen wir $\mathfrak{u}$-Wegeklassengruppe von $\mathbb{S}$ und bezeichnen mit $\mathfrak{S}_{\mathfrak{G}}(\mathfrak{u t})$. Ein Element von $\mathfrak{W}_{\mathfrak{S}}(\mathfrak{H})$ ist also eine $\operatorname{Klasse}[W(e, \alpha)]$ aller $\mathfrak{w}$-Wege $W^{\prime}(e, a)$, die mit einem bestimmten $W(e, a) \mathfrak{u}$-homotop sind. 
Nun wollen wir die Gruppe $\mathfrak{S}_{\mathscr{G}}(\mathfrak{t})$ topologisieren. Einer Umgebung $\mathfrak{H}_{0}<\mathfrak{u}$ von $e$ entsprechend definieren wir die $\mathfrak{U}_{0}$-Umgebung von $[W(e, a)]$ $\left(W(e, a)=\left\{e, a_{1}, \ldots, a_{r}, a\right\}\right)$ (der Name der Umgebung soll mit dem entsprechenden grossen Buchstabe bezeichnet werden) als die Gesamtheit aller Klassen $[W(e, a c)]$ mit $W(e, a c)=\left\{e, a_{1}, \ldots, a_{r}, a, a c\right\}, c \in \mathfrak{u}_{0}$. Dann ist $\mathfrak{S}_{\mathfrak{G}}(\mathfrak{H})$ eine topologische Gruppe. Die Prüfung, dass $\mathfrak{W}_{\mathfrak{G}}(\mathfrak{H})$ in bezug auf diese Topologie in der Tat Hausdorffscher Raum ist, bietet keine Schwierigkeit. Hier prüfen wir nur etwa das Trennungsaxiom. Es sei also $[W(e, a)] \neq\left[W^{\prime}(e, b)\right]$. Falls $a \neq b$ ist, wählen wir ut $_{0}<u$ so, dass $a 1_{0} \cap b \mathfrak{1}_{0}=0$. Dann ist $U_{0}([W(e, a)]) \cap U_{0}\left(\left[W^{\prime}(e, b)\right]\right)=0$. Wenn $a=b$ ist, gilt sogar $\mathfrak{U}([W(e, a)]) \cap \mathfrak{H}\left(\left[W^{\prime}(e, a)\right]\right)=0$, denn andernfalls müsste es ein Element $c \in \mathfrak{u}$ geben, so dass

$$
\begin{aligned}
W(e, a) \cdot\{e, c\} & =\left\{e, a_{1}, \ldots, a_{r}, a, a c\right\} \sim\left\{e, a_{1}^{\prime}, \ldots, a_{r}^{\prime}, a, a c\right\} \\
& =W^{\prime}(e, a) \cdot\{e, c\},
\end{aligned}
$$

woraus folgte

$$
\begin{aligned}
W(e, a) & =(W(e, a) \cdot\{e, c\}) \cdot\{e, c\}^{-1} \sim\left(W^{\prime}(e, a) \cdot\{e, c\}\right) \cdot\{e, c\}^{-1} \\
& =W^{\prime}(e, a)
\end{aligned}
$$

gegen der Annahme. Um nun die Stetigkeit des Produktes zu beweisen, genügt es zu zeigen, dass für eine beliebig gegebene Umgebung $\|_{0}$ eine Umgebung $\mathfrak{H}_{1}$ sich so bestimmen lässt, dass.

$$
\left[W(e, a) \cdot\{e, c\} W(e, b) \cdot\left\{e, c^{\prime}\right\}\right] \in \mathbb{H}_{0}([W(e, a) \cdot W(e, b)])
$$

für beliebige $c, c^{\prime} \in \mathfrak{t}_{1}$ gilt. Es sei

$$
W(e, a)=\left\{e, a_{1}, \ldots, a_{r}, a\right\} \text { und } W(e, b)=\left\{e, b_{1}, \ldots, b_{s}, b\right\} \text {, }
$$

und $\mathfrak{u}_{1}$ sei so gewählt, dass $b_{n} \mathfrak{u}_{0} b_{n}^{-1} \ni \mathfrak{H}_{1}(n=1, \ldots, s)$ und $b \mathfrak{u}_{0}>\mathfrak{u}_{1} b \mathfrak{u}_{1}$. Dann ist für $c^{\prime \prime}=b^{-1} c b c^{\prime}$

$$
\begin{aligned}
W(e, a) \cdot & \{e, c\} \cdot W(e, b) \cdot\left\{e, c^{\prime}\right\} \\
& =\left\{e, a_{1}, \ldots, a_{r}, a, a c, a c b_{1}, \ldots, a c b_{s}, a c b, a c b c^{\prime}\right\} \\
& \approx\left\{e, a_{1}, \ldots, a_{r}, a, a, a b_{1}, \ldots, a b_{s}, a b, a b c^{\prime \prime}\right\} \\
& =W(e, a) \cdot W(e, b) \cdot\left\{e, c^{\prime \prime}\right\}
\end{aligned}
$$

mit $c^{\prime \prime} \in \mathfrak{1}_{0}$. Die Stetigkeit des Inversen ist analog zu beweisen.

Nach O. Schreier (S. 1) heissen zwei topologische Gruppen (8), (\$) ${ }^{\prime}$ $\mathfrak{u}-\mathfrak{u}^{\prime}-$ im Kleinen topologisch isomorph, wenn zwischen den Elementen der Umgebungen $\mathfrak{u}, \mathfrak{u}^{\prime}$ der Einsen von (\$) bzw. (\$3) eine homöomorphe Zuordnung $a \leftrightarrow a^{\prime}$ derart existiert, dass 


$$
\begin{aligned}
& \text { aus } a, b, c \in \mathfrak{u}, a b=c \text { folgt } a^{\prime} b^{\prime}=c^{\prime}\left(a^{\prime}, b^{\prime}, c^{\prime} \in \mathfrak{u}^{\prime}\right) \text {, } \\
& \text { aus } a^{\prime}, b^{\prime}, c^{\prime} \in \mathfrak{u}^{\prime}, a^{\prime} b^{\prime}=c^{\prime} \text { folgt } a b=c(a, b, c \in \mathfrak{u}) .
\end{aligned}
$$

Nun ist die eineindeutige Zuordnung $[\{e, a\}] \leftrightarrow \rightarrow a, a \in \mathfrak{u}$ zwischen den Elementen der Umgebungen $\mathfrak{U}([\{e, e\}])<\mathfrak{W}_{\mathfrak{s}}(\mathfrak{u})$ und $\mathfrak{u}<\mathscr{S}$ eine homöomorphe, denn es gilt folgende eineindeutige Zuordnung für jede Umgebung $\mathfrak{u}_{0}<\mathfrak{u}$ mit $a \mathfrak{u}_{0}<\mathfrak{u}$

$$
a \mathfrak{H}_{0} \ni b \longleftrightarrow[\{e, b\}]=[\{e, a, b\}] \in \mathfrak{H}_{0}([\{e, a\}]) .
$$

Aus $a, b, a b \in \mathfrak{u}$ folgt

$$
\{e, a\} \cdot\{e, b\}=\{e, a, a b\} \approx\{e, e, a b\}=\{e, a b\},
$$

und umgekehrt, also sind $\mathfrak{S}_{\mathfrak{G}}(\mathfrak{u})$ und (5) $\mathfrak{U}$-1t-im Kleinen topologisch isomorph.

Wird im allgemeinen jeder Wegeklasse $[W(e, a)]$ das Endelement $a \in(5)$ zugeordnet, ist ersichtlich $\mathfrak{S}_{\mathfrak{S}}(\mathfrak{u})$ auf (5) stetig und gebietstreu abgebildet, falls (\$) $\mathfrak{u}$-z.h. ist. Die Untergruppe $\mathfrak{F}_{\mathfrak{G}}(\mathfrak{u})$ von $\mathfrak{W}_{\mathfrak{S}}(u)$, die aus allen Klassen der geschlossenen $\mathfrak{u}$-Wege besteht, heisse nun die $\mathfrak{u}$ Fundamentalgruppe von (5) . Ofienbar ist $F_{G}(\mathfrak{H})$ ein diskreter Normalteiler von $1 \mathrm{~s}_{\mathrm{s}}(\mathrm{u})$, und es gilt

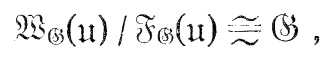

( $\cong$ bedeutet die topologische Isomorphie.) Falls (S) und u O-z.h. sind, dann ist $\mathfrak{S}_{\mathfrak{G}}(\mathfrak{H})$ anch $\mathrm{O}-\mathrm{z} . \mathrm{h}$. und $\mathfrak{F}_{\mathfrak{G}}(\mathfrak{u})$ liegt im Zentrum von $\mathfrak{S}_{\mathfrak{G}}(\mathfrak{u})$. Dies ist genau wie bei Schreier (S. 1) zu zeigen.

Wir nennen (5) u-einfach-zusammenhängend, falls alle geschlossenen u-Wege u-homotop $\mathrm{O}$ sind; und O-einfach-zusammenhängend, falls $\$$ f für jede Umgebung $\mathfrak{u}$ tt-einfach-zusammenhängend ist. Dann sieht man wie

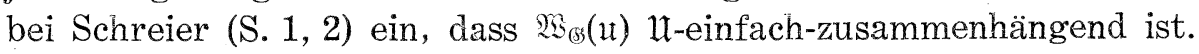

Zusammenfassend erhalten wir

Satz 1. Die $\mathfrak{u}$-Wegeklassengruppe $W_{\mathfrak{G}}(\mathfrak{u})$ von einer topologischen Gruppe (5) ist eine $\mathfrak{u}-z . h$. und $\mathfrak{u}$-einfach-zusammenhängende topologische Gruppe und mit (S) U-1t-im Kleinen topologisch isomorph. Falls (S) $\mathfrak{u}-z . h$. ist, gilt es

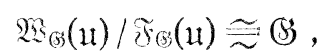

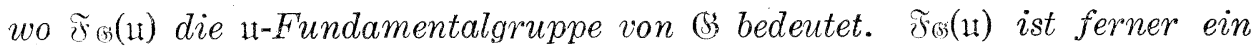
diskreter Normateiler von $W_{\mathfrak{G}}(\mathfrak{u t})$; es ist sogar im Zentrum von $W_{\mathfrak{G}}(\mathfrak{u})$ enthalten, falls (5) uud u 0 -z.h. ist.

Wegen einer späteren Anwendung sei hier noch der folgende Satz angefuhrt: 
Satz 2. Ist (S) kompakt metrisch und u zusammenhängend, so besitzt $\widetilde{\mho}_{\mathfrak{G}}(\mathfrak{u})$ nur endlich viele Erzeugende.

Beweis. Aus der Voraussetzung folgt, dass es für jeden $u$-Weg einen damit $\mathfrak{u}$-homotopen $\mathfrak{b}$-Weg mit $\mathfrak{b}^{2}<\mathfrak{u}$ gibt. Da ferner (s) kompakt metrisch ist, gibt es endlich viele Elemente $e, a_{2}, \ldots, a_{r} \in \mathbb{S}$ derart, dass $\mathfrak{v}, a_{2} \mathfrak{b}, \ldots, a_{r} \mathfrak{b}$ ganz (5) überdecken. Wir zeigen nun, dass die höchstens $r^{2 r} \mathfrak{u}$-Wege

$$
W\left(i_{1}, \ldots, i_{2 r}\right)=\left\{e, a_{i_{1}}, \ldots, a_{i_{2 r}}, e\right\}
$$

die Gruppe $\tilde{F}_{\mathscr{S}}(\mathfrak{u})$ erzeugen. Es sei also $W=\left\{e, b_{1}, \ldots, b_{s}, e\right\}$ ein beliebiger geschlossener $\mathfrak{b}$-Weg. Dann gibt es einen damit $\mathfrak{b}$-homotopen $\mathfrak{v}^{2}-$ Weg

$$
W^{\prime}=\left\{e, a_{j_{1}}, \ldots, a_{j_{s}}, e\right\}
$$

mit $a_{j_{2 h}} \mathfrak{v} \ni b(n=1, \ldots, s)$; wir , reduzieren “ dann $W^{\prime}$ so, dass die benachbarten Elemente voneinander verschieden sind, und bezeichnen es wieder mit (3). Falls $s \geqq r$ ist, gibt es mindestens zwei gleiche Elemente unter $e, a_{j_{1}}, \ldots, a_{j_{s}}$. Das früheste Paar sei $a_{j_{u}}=a_{i_{v}}, u<v-1$. Dann ist $W^{\prime}=W_{1} \cdot W_{2}$ mit $W_{1}=\left\{e, a_{j_{1}}, \ldots, a_{j_{u}}, \ldots, a_{j_{v}}=a_{j_{u}}, a_{j_{u-1}}, \ldots, a_{j_{1}}, e\right\}$ und $W_{2}=\left\{e, a_{j_{1}}, \ldots, a_{j_{u}}=a_{j_{v}}, a_{j_{v+1}}, \ldots, a_{j_{s}}, e\right\}$. Nach der Voraussetzung über $a_{j_{v}}$ sind aber alle $e, a_{j_{1}}, \ldots, a_{j_{v-1}}$ von einander verschieden; also ist die Elementenanzahl von $W_{1} \leqq 2 r$. Da die Elementenanzahl von $W_{2}$ sicher kleiner als die von $W_{1}$ ist, erbringen wir nach höchistens $s$ Schritten eine Zerlegung von $W^{\prime}$ in folgende Gestalt:

$$
W^{\prime} \sim W\left(i_{1}^{(1)}, \ldots, i_{2 r}^{(1)}\right) \cdots W\left(i_{1}^{(p)}, \ldots, i_{2 r}^{(p)}\right)\left(\mathfrak{b}^{2}\right) .
$$

2. Nun definieren wir nach Schreier (S. 1) die 1t-universelle Utberlagerungsgruppe $\ddot{U}_{\mathscr{S}}(\mathfrak{u})$ von $(\mathrm{S}$ folgeddermassen. Es sei th eine Umgebung von $e$ mit $\mathfrak{u}=\mathfrak{H}^{-1}$. Jedem Element $a \in \mathfrak{t}$ entsprechend führen wir abstrakt ein Element $A$ ein, und jeder Relation $a \cdot b=c, a, b, c \in \mathfrak{u}$ ordnen wir eine Relation $A \cdot B \cdot C^{-1}=1$ zwischen entsprechenden Elementen zu. Wir definieren dann $\ddot{U}_{6}(11)$ als Gruppe mit den Erzeugenden $\{A\}$ und mit den definierenden Relationen $A \cdot B \cdot C^{-1}=1$. Für eine Umgebung: $\mathfrak{H}_{0} \subset \mathfrak{u}$ in $\mathbb{S}$ definieren wir die $\mathfrak{H}_{0}$-Umgebung $U_{0}\left(A_{1} \ldots A_{r}\right), A_{1} \ldots A_{r} \in \ddot{\mathbb{U}}_{6(\mathfrak{H}}(\mathfrak{u})$ als Gesamtheit aller Elemente $A_{1} \ldots A_{1} B$ mit $B \longleftrightarrow b \in \mathfrak{1}_{0}$. Dann wird $\ddot{U}_{6}(u)$ eine topologische Gruppe. Ist (3) H-z.h., dann ist auch $\mathfrak{d}_{G}(\mathfrak{u}) \mathfrak{U}-z . h$. Ferner wird $\dot{\mathbb{U}}_{6}(\mathfrak{u})$ auf $\left(\mathbb{S}\right.$ durch $A_{1} \ldots A_{r} \rightarrow a_{1} \ldots a_{r}\left(a_{i} \in \mathfrak{u}\right) \mathfrak{H}-\mathfrak{H}(E)$-im Kleinen topologisch isomorph abgebildet (Vgl. Schreier (S. 1)) .

Eine grundlegende Eigenschaft von $\ddot{U}_{(s)}(u)$ ist die, dass für jede $u-z \cdot h$. topologische Gruppe $\mathbb{G S}^{\prime}$, die mit $(5) \mathfrak{u}-\mathfrak{u}$-im Kleinen topologisch isomorph ist, sich ein diskreter Normalteiler $\mathfrak{U} \subset \ddot{U}_{\mathfrak{s}}(\mathfrak{H})$ so finden lässt, dass 


$$
\ddot{\mathbb{U}}_{\mathscr{S}}(\mathfrak{H}) / \mathfrak{A} \cong \mathbb{S}^{\prime}
$$

gilt. Ist dazu noch $\left(\mathfrak{S}\right.$ O-z.h., dann ist $\mathfrak{Y}$ im Zentrum von $\ddot{\mathbb{U}}_{\mathfrak{G}}(\mathfrak{l t})$ enthalten (S. 1).

Nun können wir $\ddot{U}_{\mathfrak{G}}(\mathfrak{t})$ als eine Art der $\mathfrak{t}$-Wegeklassengruppe auffassen. Schreiben wir nämlich für zwei u-Wege $W(e, a)=\{e, \ldots, s, s p$, $s p q, t, \ldots, a\}(p, q \in \mathfrak{u})$ und $W^{\prime}(e, a)=\{e, \ldots, s, s p q . t, \ldots, a\}$

$$
W(e, a) \equiv W^{\prime}(e, a)(\mathfrak{u}) \text {, }
$$

falls auch $p q$ in $\mathfrak{u}$ enthalten ist. Wenn für zwei $\mathfrak{u}$-Wege $W$ und $W^{\prime}$

$$
W \equiv W_{1} \equiv W_{2} \equiv \cdots \equiv W_{r} \equiv W^{\prime}(\mathfrak{u})
$$

mit $\mathfrak{u}$-Wegen $W_{n}(n=1,2, \ldots, r)$ gilt, dann sagen wir : $W$ sei mit $W^{\prime}$ u-verwandt, und bezeichnen es mit

$$
W \equiv W^{\prime}(\mathrm{u}) \text {. }
$$

Die $\mathfrak{u}-W$ ege $W$ aus $\left.\mathfrak{S}_{\mathscr{S}}^{0} \mathfrak{h}\right)$ mit $W \equiv\{e, e\}(\mathfrak{t})$ bilden dann offenbar einen Normalteiler $\mathfrak{N}^{1}$ von $\mathfrak{S}_{\mathfrak{G}}^{0}(\mathfrak{u})$. Ein Element von $\mathfrak{S}_{\mathfrak{G}}^{0}(\mathfrak{H}) / \mathfrak{N}^{1}$ ist also eine Klasse der miteinander 1t-verwandten 1 -Wege. Wir topologisieren nun $\mathfrak{S}_{\mathscr{G}}^{0}(\mathfrak{t}) / \mathfrak{Z}^{1}$ in derselben Weise wie für $\mathfrak{S}_{\mathfrak{G}}(\mathfrak{H})=\mathfrak{S}_{\mathfrak{B}}^{0}(\mathfrak{u}) / \mathfrak{R}^{0}$. Dann sehen wir sofort ein, dass die Zuordnung

$$
W\left(e, a_{1}, \ldots, a_{r}\right)=\left\{e, a_{1}, a_{1} a_{2}, \ldots, a_{1} \ldots a_{r}\right\} \rightarrow A_{1} \ldots A_{r},\left(a_{n} \in \mathfrak{u}\right)
$$

$\mathfrak{S B}_{\mathscr{G}}(\mathfrak{u}) / \mathfrak{N}^{1}$ auf $\ddot{\mathfrak{U}}_{\mathscr{G}}(\mathfrak{u})$ topologisch isomorph abbildet:

$$
\ddot{U}\left(\mathfrak{s}(\mathfrak{H}) \cong \mathfrak{G}_{(j)}^{0}(\mathfrak{t}) / \Re^{1}\right. \text {. }
$$

Erisichtlich ist $\ddot{\mathbb{U}}_{\mathfrak{s}}(\mathfrak{u}) \mathfrak{U}$-einfach-zusammenhängend, denn die $\mathfrak{u}$-Wegeklassengruppe von $\ddot{\mathfrak{U}}_{\mathfrak{G}}(u)$ stimmt (nach Satz 1) nach der oben erwähnten Grundeigenschaft von $\ddot{\mathcal{U}}_{\mathrm{s}}(\mathrm{u})$ mit $\ddot{\mathrm{U}}_{\mathrm{s}}(\mathrm{u})$ überein. Ebenso gilt

$$
\ddot{U}_{G}(\mathfrak{u}) / \mathfrak{U} \cong \mathfrak{M}_{G}(\mathfrak{u}),
$$

dabei ist $\mathfrak{U}$ die Gesmtheit aller $\mathfrak{u}$-verwandten Wegeklassen, die $\mathfrak{t}$-homotop $\mathrm{O}$ sind. Dass $\mathfrak{A} \mathrm{im}$ allgemeinen nicht gleich der Einsgruppe ist, zeigt das folgende Beispiel.

Beispiel 1. Es sei $G$ die additive Grnppe der modulo 1 reduzierten reellen Zahlen. Als Umgebung der Eins sei $\mathfrak{u}=\{s\}\left(-\frac{1}{4}-\varepsilon<s<\frac{1}{4}+\epsilon\right)$ für ein genügend kleines $\varepsilon>0$ gewählt, und es sei $a=\frac{1}{8}$. Dann ist

$$
\{e, a, 3 a, 5 a, 7 a, e\} \approx\{e, a, a,-a,-a, e\} \approx\{e, e\}(\mathfrak{t}) \text {, }
$$


aber nicht $\equiv\{e, e\}$, weil die Relation $8 a=e$ nicht aus Relationen in $\mathfrak{u}$ zusammengesetzt werden kann.

Da $\mathfrak{S}_{\mathfrak{G}}(\mathfrak{H})$ und $\ddot{\mathfrak{U}}_{\mathfrak{G}}(\mathfrak{H})$ beide $\mathfrak{u}$-einfach-zusammenhängend sind, ist also $\ddot{U}_{\text {os }}(u)$ nicht eindeutig durch die Eigenschaft, bestimmt, dass es $\mathfrak{u}$-einfachz.h. und $\mathfrak{t}$ - $\mathfrak{A}$-im Kleinen topologisch isomorph mit $(\mathbb{3}$ sein soll. Es gilt aber

Satz 3. Eine $\mathfrak{u}^{\prime}-z . h$. und $\mathfrak{u}^{\prime}$-einfach-zusammenhängende topologische Gruppe (S', die mit (S) $\mathfrak{u}^{\prime}-\mathfrak{l}$-im Kleinen topologisch isomorph ist, ist dann und nur dann mit $\ddot{U}_{\mathfrak{G}}(\mathrm{u})$ im Grossen topologisch is morph, ween (\$) ausserdem mit $\ddot{U}_{\mathfrak{G}}(\mathfrak{u}) \mathfrak{u}_{1}^{\prime}-\mathfrak{u}_{1}-i m$ Kleinen topologisch isomorph ist, wobei $\mathfrak{u}_{1}>\mathfrak{u}^{2}$, $\mathfrak{U}_{1}>\mathfrak{u}^{2}$ sein soll.

Der Beweis defür lässt sich ohne besondere Scnwierigkeiten aus der Grundeigenschaft von $\ddot{U}_{\mathfrak{G}}(\mathfrak{u})$ ausgeführt.

3. Hilfsatz. In jeder topologischen Gruppe gilt folgendes für beliebige zwei $\mathfrak{u}$-Wege $W_{1}, W_{2}$ und für jede Umgebung $\mathfrak{u}$ mit $\mathfrak{u}=\mathfrak{u}^{-1}$ :

$$
\begin{array}{llll}
\text { aus } & W_{1} \equiv W_{2}(\mathfrak{u}) \text { folgt } & W_{1} \sim W_{2}(\mathfrak{u}), \\
\text { aus } & W_{1} \sim W_{2}(\mathfrak{u}) \text { folgt } & W_{1} \equiv W_{2}\left(u^{2}\right) .
\end{array}
$$

Beweis. (4) folgt. direkt aus Definitionen von $\equiv$ und $\sim$ (u) . Beim Beweis von (5) kann man sich auf den Fall

$$
W_{1} \approx W_{2}(\mathfrak{u})
$$

beschränken, wobei $W_{1}=\left\{e, a_{1}, \ldots, a_{r}\right\}, W_{2}=\left\{e, a_{1}^{\prime}, \ldots, a_{r-1}^{\prime}, a_{r}\right\}$ mit $a_{i}^{-1} a_{i}^{\prime} \in \mathfrak{u}$ ist. Es gelte nun

$$
\begin{aligned}
\left\{e, a_{1}, \ldots, a_{n}, a_{n}, a_{n+1}, \ldots, a_{r}\right\} & \\
& \equiv\left\{e, a_{1}^{\prime}, \ldots, a_{n}^{\prime} . a_{n}, a_{n+1}, \ldots, a_{r}\right\}\left(\mathfrak{u}^{2}\right),
\end{aligned}
$$

dann ist für $a_{n}^{-1} a_{n}^{\prime}=c_{n}, a_{n}^{-1} a_{n+1}=d_{n}$ und $a_{n}^{\prime-1} a_{n+1}^{\prime}=d_{n}^{\prime}$ aus $\mathfrak{u}$

$$
\begin{aligned}
&\left\{e, \ldots, a_{n}, a_{n}, a_{n+1}, \ldots, a_{r}\right\} \equiv\left\{e, a_{1}^{\prime}, \ldots, a_{n}^{\prime}, a_{n}, a_{n+1}, \ldots, a_{r}\right\} \\
& \quad=\left\{e, a_{1}^{\prime}, \ldots, a_{n}^{\prime}, a_{n}^{\prime} c_{n}^{-1}, a_{n}^{\prime} c_{n}^{-1} d_{n}, a_{n+2}, \ldots, a_{r}\right\} \\
& \equiv\left\{e, a_{1}^{\prime}, \ldots, a_{n}^{\prime}, a_{n}^{\prime}, a_{n}^{\prime} c_{n}^{-1} d_{n}, a_{n+2}, \ldots, a_{r}\right\}\left(\mathfrak{u}^{2}\right) \\
&=\left\{e, a_{1}^{\prime}, \ldots, a_{n}^{\prime}, a_{n}^{\prime}, a_{n}^{\prime} d_{n}^{\prime} c_{n+1}^{-1}, a_{n+2}, \ldots, a_{r}\right\} \\
& \equiv\left\{e, a_{1}^{\prime}, \ldots, a_{n}^{\prime}, a_{n}^{\prime} d_{n}^{\prime}, a_{n}^{\prime} d_{n}^{\prime} c_{n+1}^{-1}, a_{n+2}, \ldots, a_{r}\right\}\left(\mathfrak{u}^{2}\right) \\
&=\left\{e, a_{1}^{\prime}, \ldots, a_{n}^{\prime}, a_{n+1}^{\prime}, a_{n+1}, a_{n+2}, \ldots, a_{r}\right\}
\end{aligned}
$$

mit $a_{n}^{\prime-1} a_{n+1}=d_{n}^{\prime} c_{n \div 1}^{-1}=c_{n}^{-1} d_{n} \in \mathfrak{u}^{2}$. Man zeigt also (6) durch vollständige Induktion. 
Aus (4) folgt die schon oben erwähnte Relation

$$
\ddot{\mathbb{U}}_{\mathscr{S}}(\mathfrak{u}) / \mathfrak{U} \cong \mathfrak{S}_{\mathscr{S}}(\mathfrak{u}) \text {, }
$$

also gibt es eine stetige und gebietstreue Abbildung $\rho$ von $\ddot{\mathbb{U}}_{\mathscr{S}}(\mathfrak{h})$ auf $\mathfrak{S}_{\mathfrak{G}}(u)$. Aus (5) folgt andererseits

$$
\mathfrak{S}_{\mathfrak{G}}(\mathfrak{b}) / \mathfrak{B} \cong \ddot{\mathbb{U}}_{\mathfrak{G}}(\mathfrak{H})
$$

für $\mathfrak{v}^{2}<\mathfrak{u}$, falls $\mathfrak{u} \mathfrak{v}$-z.h. ist. Denn es gibt dann für jeden $\mathfrak{u}$-Weg $W$ auf (5) einen damit $\mathfrak{u}$-homotopen $\mathfrak{v}$-Weg. Es gibt also eine stetige und gebietstreue Abbildung $\psi$ von $\mathfrak{S}_{\mathfrak{G}}(\mathfrak{b})$ auf $\ddot{U}_{\mathfrak{G}}(\mathfrak{u})$.

Von nun an nehmen wir an: (5) sei O-z.h. und im Kleinen O-z.h., und genüge dem 1. Abzählbarkeitsaxiom. Dann können wir ein abzählbares Umgebungssystem $\left\{\mathfrak{H}_{n}\right\}(n=1,2, \ldots)$ von $e$ so bilden, dass $\mathfrak{i}_{n}=\mathfrak{H}_{n}^{-1}$, $\mathfrak{u}_{n} \subset \mathfrak{u}_{n-1}$, jedes $\mathfrak{u}_{n}$ O-z.h. ist, und dass $\left\{\mathfrak{u}_{n}\right\}$ mit dem System aller Umgeoungen von $e$ äquivalent ist. Da jede $\mathfrak{u}_{n}$-Wegeklasse in einer $\mathfrak{1}_{n-1}$-Wegeklasse enthalten ist, und da es umgekehrt für jeden $\mathfrak{u}_{n-1}$-Weg $W$ einen solchen $\mathfrak{u}_{n}$-Weg gibt, der mit $W \mathfrak{u}_{n-1}$-homotop ist, so ergibt sich, dass $\mathfrak{S}_{\mathscr{G}}\left(\mathfrak{u}_{n}\right)$ auf $\mathfrak{B}_{\mathfrak{S}}\left(\mathfrak{u}_{n-1}\right)$ homomorph, stetig und gebietstreu abgebildet werden kann. Also können wir die $\mathbb{S}_{n}$-adische Limesgruppe( ${ }^{7}$ ) der Folge $\left\{\mathbb{S}_{n}\right\}, \mathbb{S}_{n}=\mathfrak{W}_{\mathbb{S}}\left(\mathfrak{u}_{n}\right)$ bilden. Wir nennen sie die O-Wegeklassengruppe und bezeichnen mit $\mathfrak{S}_{\mathfrak{G}}(0)$. Insbesondere enthält $\mathfrak{S}_{\mathfrak{G}}(0)$ die Vietorissche Fundamentalgruppe $\mathfrak{F}_{5}(0)$ von $\mathbb{S}$ : die $\mathbb{S}_{n}$-adische Limesgruppe der Folge $\mathfrak{F}_{\mathfrak{G}}\left(\mathfrak{u}_{n}\right)$. $\mathfrak{F}_{\mathscr{S}}(0)$ ist offenbar O-dimensional und im Zentrum von $\mathfrak{S}_{\mathfrak{G}}(0)$ enthalten. Es ist leicht zu sehen, dass $\mathfrak{B}_{\mathfrak{B}}(0)$, ebenso wie $\mathfrak{F}_{\mathscr{G}}(0)$, von der Wahl von $\left\{\mathfrak{u}_{n}\right\}$ unabhängig durch (5) allein bestimmt ist, und es gilt

$$
\mathfrak{W}_{\mathfrak{G}}(0) / \mathfrak{F}_{\mathfrak{G}}(0) \cong \mathbb{S} .
$$

Satz 4. Die O-Wegeklassengruppe $\mathfrak{S}_{\mathrm{S}}(0)$ lässt sich auch als $\mathbb{S}_{n}$-adishce Limesgruppe der Folge $\left\{\ddot{\mathrm{U}}_{\mathrm{s}}\left(\mathrm{u}_{n}\right)\right\}$ auffassen.

Beweis. Die genannte $\mathbb{S}_{n}$-adische Limesgruppe $\ddot{\mathbb{U}}_{\mathscr{S}}(0)$ ist offenbar von der Wahl von $\left\{\mathfrak{u}_{n}\right\}$ unabhängig. Nun sei also o.B.d.A. $\mathfrak{u}_{n}$ so gewählt, dass $\mathfrak{u}_{n}^{2}<\mathfrak{H}_{n-1}$ ist. Die folgenden homomorphen, stetigen und gebietstreuen Abbildungen

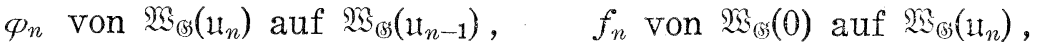

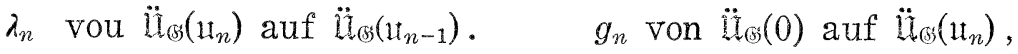

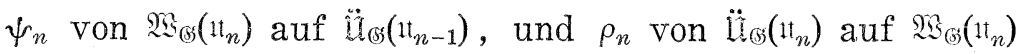

genügen den Relationen

(7) Vgl. H. Freudenthal, a.a. O. (2), S. 170. 


$$
\psi_{n} \rho_{n}=\lambda_{n}, \rho_{n-1} \psi_{n}=\phi_{n}, \varphi_{n} f_{n}=f_{n-1} \quad \text { and } \quad \lambda_{n} g_{n}=g_{n-1} .
$$

Nun gilt für die homomorphen, stetigen und gebietstreuen Abbildungen $\rho_{n} g_{n}$ von $\ddot{U}_{G}(0)$ auf $\mathfrak{S}_{6}\left(\mathrm{ti}_{n}\right)$

$$
\varphi_{n}\left(\rho_{n} g_{n}\right)=\left(\rho_{n-1} \psi_{n}\right)\left(\rho_{n} g_{n}\right)=\rho_{n-1}\left(\lambda_{n} g_{n}\right)=\rho_{n-1} g_{n-1},
$$

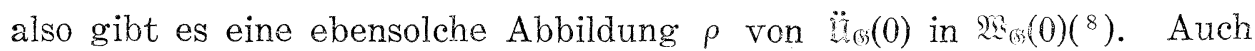
umgekehrt gilt für die homomorphen, stetigen und gebietstreuen $\mathrm{Ab}$ biidungen $\psi_{n+1} f_{n: 1}$ von $\underline{s}_{6}(0)$ auf $\tilde{U}_{6 s}\left(1 t_{n}\right)$

$$
\lambda_{n}\left(\psi_{n+1} f_{n+1}\right)=\left(\psi_{n} \rho_{n}\right)\left(\psi_{n+1} f_{n+1}\right)=\psi_{n}\left(\rho_{n} f_{n+1}\right)=\psi_{n} f_{n},
$$

also gibt es wieder eine ebensolche Abbilding $\psi$ von $\widetilde{\mho}_{\mathscr{\sigma}}(0)$ in $\hat{\mathbb{U}}_{\mathrm{g}}(0)$. Da aber $\rho \psi=1$ bzw. $\psi \rho=1$ in $\mathfrak{S}_{G}(0)$ bzw. $\ddot{U}_{\mathfrak{G}}(0)$ gilt, ist $\rho=\psi^{-1}$ bzw. $\psi=\rho^{-1}$ in $\mathfrak{B}_{\mathfrak{G}}(0)$ bzw. $\ddot{\mathfrak{d}}_{\mathfrak{s}}(0)$, d.h. es gilt

$$
\mathfrak{S}_{\mathrm{G}}(0) \cong \ddot{U}_{\mathrm{S}}(0) \text {. }
$$

Satz 5. Für jede mit (5) im Kleinen topologisch isomorphe O-zusammenhängende topologische Gruppe (\$)' gilt

$$
\left(\mathfrak{S}^{\prime} \cong \mathfrak{S}_{\mathscr{B}}(0) / \mathfrak{A},\right.
$$

wo $\mathfrak{X}$ ein nulldimensionaler, abgeschlossener, im Zentrum von $\mathfrak{B}_{\mathfrak{S}}(0)$ enthaltener Normalteiler von $\mathfrak{B}_{(x)}(0)$ ist.

Beweis. (S') sei mit (S) $\|^{\prime}-1 t$-im Kleinen topologisch isomorph, dann ist es auch mit (\$) $11_{n}^{\prime}-1 t_{n}$-im Kleinen topologisch, isomorph, wenn $1_{n}<n$ ist. Für ein solches $"_{n}$ gilt also nach der Grundeigenschft von $\ddot{\mathbb{U}}_{6}\left(\|_{n}\right)$

$$
\mathfrak{G}^{\prime} \cong \dot{U}_{6}\left(1 t_{n}\right) / \mathfrak{A}_{n} \text {, }
$$

wo $\mathfrak{U}_{n}$ ein diskreter Normalteiler des Zentrums von $\ddot{\mathrm{U}}_{6}\left({ }_{1 t_{n}}\right)$ ist. Nach Satz 5 gibt es aber einen nulldimensionalen abgeschlossenen Normalteiler $\mathfrak{B}_{n}<\mathfrak{N}_{\mathfrak{S}}(0)$ mit

$$
\mathfrak{S}_{G}(0) / \mathfrak{B}_{n} \cong \ddot{\mathfrak{U}}_{\Theta}\left(\mathrm{it}_{n}\right) \text {. }
$$

Aus (10) und (11) folgt der Satz.

Satz 6. $\mathfrak{S}_{\mathrm{G}}(0)$ ist O-z.h., im Kleinen O-z.h, und O-einfachzusammenhängend.

Beweis. Da $\mathfrak{S}_{\mathfrak{S}}(0)$ die Limesgruppe von $\left\{\mathfrak{E}_{\mathbb{G}}\left(\mathrm{lt}_{n}\right)\right\}$ mit O.-zusammenhängenden $1_{n}$ ist, kann jedes Element von $\mathfrak{B E}_{6}(0)$ durch

$$
\left(W_{1}, W_{2}, \ldots, W_{n}, \ldots\right)
$$

(8) Vgl. H. Freudenthal, a.a. O. (2), S. 157, Dritter Limessatz. 
mit $\varphi_{n} W_{n}=W_{n-1}, \quad W_{n} \in \mathfrak{W}_{\mathfrak{B}}\left(\mathrm{Ht}_{n}\right)$ dargestellt werden. Wir bezeichen nun mit $\mathfrak{U}_{m}^{(n)}$ dis Gesamtheit aller Elemente von $\mathfrak{S}_{\mathbb{G}}(0)$, deren $n$-ten Komponenten $W_{n}$ in der mit $t_{m} \in \mathbb{3}$ topologisch isomorphen Umgebung $\mathfrak{U}_{m}(m=n, n+1, \ldots)$ von $\mathfrak{S}_{\mathscr{m}}\left(\mathfrak{H}_{n}\right)$ enthalten sind. Dann bildet $\left\{\mathfrak{U}_{m}^{(n)}\right\}$ $(n=1,2, \ldots ; m=n, n+1, \ldots)$ ersichtlich ein vollständiges Umgebungssystem der Eins von $\mathfrak{S}_{6}(0)$. Man kann nun setzen $\mathfrak{W}_{65}(0) / \mathfrak{N}_{n} \cong \mathfrak{Q}_{65}\left(\mathfrak{1}_{n}\right)$. Dass $\mathfrak{S}_{\mathfrak{s}}(0) \mathfrak{U}_{m}^{(n)}$-z.h. bzw. $\mathfrak{U}_{m}^{(n)}$-einfach-zusammenhängend ist, folgert man leicht daraus, dass $\mathfrak{S}_{\mathbb{S}}\left(\mathfrak{i t}_{n}\right) \mathfrak{U}_{m}$-z.h. bzw. $\mathfrak{U}_{m}$-einfachzusammenhängend ist, indem man die Faktorgruppe nach $\mathfrak{N}_{n}$ betrachtet. Also ist $\mathfrak{S}_{\mathbb{S}}(0)$ O-z.h. und O-einfach-zusammenhängend. Dass $\mathfrak{S}_{\mathfrak{G}}(0)$ im Kleinen O-z.h. ist, ist ganz analog zu beweisen.

Wir nennen eine topologische Gruppe (\$) im Kleinen O-einfach-zusammenhängend, wenn es in (5) eine Umgebung $\mathfrak{v}$ der Eins mit der Eigenschaft gibt, dass für jede Umgebung $\mathfrak{t}<\mathfrak{v}$ jeder geschlossene $\mathfrak{t}-W$ eg in $\mathfrak{v} \mathfrak{t -}$ homotop $\mathrm{O}$ ist.

Dann lässt sich wie üblich zeigen, dass für eine O-z.h., im Kleinen O-z.h. topologische Gruppe (5)

$$
\mathfrak{W}_{G}(0)=\mathfrak{W}_{\mathscr{G}}\left(\mathfrak{t}_{n}\right) \quad \text { (für.r genügend grosses } n \text { ) }
$$

dann und nur dann gilt, wenn (5) dazu noch im Kleinen O-einfach-z.h. ist. Im allgemeinen ist also $\mathfrak{S}_{\mathfrak{G}}(0)$ nicht mit $\$ \mathrm{im}$ Kleinen topologisch isomorph.

Beispiel 2. (5) sei das direkte Produkt abzählbar unendlich vieler additiver Gruppen $\mathscr{S}_{0}$ der modulo 1 reduzierten reellen Zahlen. Die Umgebung $\mathfrak{t t}_{n}$ sei die Gesamtheit aller Elemente, deren ersten $n$-Komponenten $a_{i}(i=1 . \ldots, n)\left|a_{i}\right|<\frac{1}{n}$ genügeu. Dann ist

$$
\mathfrak{W}_{\mathfrak{G}}\left(\mathfrak{u}_{n}\right)=\overbrace{\mathfrak{G}_{1} \times \cdots \times \mathfrak{G}_{1}}^{n} \times \mathbb{S}_{0} \times \mathfrak{G}_{0} \times \cdots,
$$

wobei $\mathfrak{S}_{1}$ die additiven Gruppe der reellen Zahlen bedeutet. Also ist

$$
\mathfrak{S}_{\mathfrak{G}}(0)=\mathfrak{S}_{1} \times \mathbb{S}_{1} \times \mathbb{S}_{1} \times \ldots,
$$

und dies ist nicht mit (S) im Kleinen topologisch isomorph.

Wir erhalten ferner leicht folgende Sätze.

Satz 7. $\mathfrak{s}_{\omega_{5}}(0)$ ist dann und nur dann abelsch, wenn (5) abelsch ist.

Satz 8. Das direkte Produkt $\mathrm{G}_{1} \times \mathbb{S}_{2}$ von zwei O-zusammenhängenden, im Kleinen O-zusammeenhöngenden topologischen Gruppen $\mathbb{S}_{1}, \mathbb{S}_{2}$ ist auch $O-z . h .$, und im Kleinen O-z.h., und und es gilt

$$
\begin{aligned}
& \mathfrak{W}_{\mathfrak{G}_{1} \times \mathfrak{G}_{2}}(0) \cong \mathfrak{W}_{\mathfrak{G}_{1}}(0) \times \mathfrak{W}_{\mathfrak{G}_{2}}(0), \\
& \widetilde{\mho}_{\mathfrak{G}_{1} \times \mathfrak{G}_{2}}(0) \cong \mathfrak{F}_{\mathfrak{G}_{1}}(0) \times \widetilde{F}_{\mathfrak{G}_{2}}(0) .
\end{aligned}
$$


Satz. 9. Dann und nur dann ist $\mathfrak{S}_{\mathfrak{S}}(0)$ vollständig bzw. im Kleinen kompakt bzw. komplettierbar falls (3) vollständig bzw. im Kleinen kompakt bzw. komplettierbar ist. Im letzten Fall ist

$$
\mathfrak{S}_{\mathbb{G}} k(0) \cong\left(\mathfrak{M}_{\mathbb{G}}(0)\right)^{k},
$$

wo $k$ die Komplettierung bedeutet.

\section{$\S 2$. Stetige projektive Darstellung.}

1. Es sei $\Re_{n}$ die volle lineare Gruppe $n$-ten Grades, nämlich die multiplikative Gruppe aller Matrizen $A=\left(a_{i j}\right)$ von dem Grade $n$ und mit der nicht verschwindenden Determinante: $|A| \neq 0$, deren Komponenten $a_{i j}$ zum komplexen Zahlkörper $\Re$ gehören. Sei ferner $\partial_{n}$ das Zentrum von $\mathfrak{\Omega}_{n}$, also die Gesamtheit aller Matrizen $\alpha E_{n}, \alpha \in \Re$, wo $E_{n}$ die Einheitsmatrix bedeutet. Wir topologisieren $\mathfrak{N}_{n}$ wie gewöhnlich mit der Metrik

$$
\rho_{0}(A, B)=\|A-B\|, \quad\|A\|=\sqrt{\sum_{i, j=1}^{n}\left|a_{i j}\right|^{2}},
$$

und nennen die Gruppe

$$
\mathfrak{P}_{n}=\mathfrak{\Re}_{n} / \Re_{n}
$$

nach der üblichen Topologie der Faktorgruppe die projektive Gruppe nten Grades. Wir bezeichnen mit $\bar{A}$ die Klasse mod. $\Omega_{n}$ von $A$ oder das Element von $\mathfrak{F}_{n}$, das $A$ entspricht.

Es sei nun $\mathfrak{U}_{n}$ die spezielle lineare Gruppe, nämlich die Untergruppe von $\Re_{n}$, die durch $|A|=1$ charakterisiert wird. Dann ist

$$
3_{n}^{0}=3_{n} \cap \mathfrak{U}_{n}=\left\{\omega E_{n}\right\}\left(\omega^{n}=1\right)
$$

das Zentrum von $\mathfrak{u}_{n}$, und es gilt

$$
\mathfrak{F}_{n}=\mathfrak{A}_{n} / 3_{n}=\mathfrak{U}_{n} \vee B_{n} / 3_{n} \cong \mathfrak{U}_{n} / 8_{n} \cap \mathfrak{U}_{n}=\mathfrak{H}_{n} / 3_{n}^{0}\left({ }^{9}\right)
$$

Indem wir also jedes Element von $\Re_{n}$ durch die Klasse $\tilde{A}$ mod. $\beta_{n}^{0}$ von $\mathfrak{U}_{n}$ darstellen, können wir folgende Metrik $\rho_{1}$ von $\mathfrak{F}_{n}$ einführen:

$$
\rho_{1}(\widetilde{A}, \widetilde{B})=\operatorname{Min}_{i=1, \cdots, n}\left(\rho_{0}\left(A, B \omega^{i}\right)\right)=\operatorname{Min}_{i=1, \cdots, n}\left(\rho_{0}\left(A \omega^{i}, B\right)\right) .
$$

Die hierdurch definierte Topologie ist offenbar mit der früher definierten äquivalent.

(9) H. Freudenthal, Einige Satze über topologische Gruppen, Annals of Math., 37 (1936), S. 50. 
Definition 1. $\left({ }^{10}\right)$ Die stetige projektive Darstellung (S.P.D.) 3 von (3) ist die homomorphe stetige Abbildung von (S) in $\mathfrak{F}_{n}$. Also:

1) 5 ordnet jedem Element $a \in \mathbb{S}$ eindeutug ein Element $\overline{D(a)}$ aus $\mathfrak{S}_{n}$ zu, und es gilt dabei:

$$
\overline{D(a)} \cdot \overline{D(b)}=\overline{D(a b)}, \quad a, b \in \mathbb{S},
$$

3) die Zuordnung $a \rightarrow \overline{D(a)}$ ist stetig.

Zwei S.P.D. $\overline{2}_{1}$ und $\overline{2}_{2}$ heissen aquivalent, wenn

$$
\bar{P} \overline{D_{1}(a)} \bar{P}^{-1}=\overline{D_{2}(a)} \text {, für jedes } a \in \mathbb{S}^{5}
$$

mit einem Element $\bar{P} \in \mathfrak{F}$ gilt, und $\bar{D}$ heisst irreduzibel bzw. vollständig reduzibel, wenn die Matrizenmenge $\{\alpha D(\alpha)\}, \alpha \in \Re$ für alle Elemente $a \in(S)$ irreduzibel bzw. vollständig reduzibel ist.

Wählen wir auf irgendeine Weise einen Repräsentanten $D(\alpha)$ aus der Klasse $\overline{D(a)}$, dann gilt

$$
D(a) \cdot D(b)=u_{\alpha, b} D(a b), \quad \text { mit } u_{a, b} \in \Re .
$$

Aus dem Assoziativgesetz folgt

$$
u_{a, b} u_{a b, c}=u_{a, b c} u_{b, c} .
$$

Wir nennen $u_{a, b}$ ein 5 zugehöriges Faktorensystem. Wenn wir statt $D(a)$ einen anderen Repräsentanten $D(a)^{\prime}=a_{a} D(a)$ aus $\overline{D(a)}$ wählen, dann gilt

$$
u_{a, b}^{\prime}=\frac{\alpha_{a} \alpha_{b}}{\alpha_{a b}} u_{a, b}
$$

Solche Faktorensysteme $u_{a, b}$ und $u_{a, b}^{\prime}$ nennen wir assoziiert. Einer S.P.D. 3 entspricht dann eindeutig eine Klasse assiziierter Faktorensysteme $\left\{u_{\alpha, b}\right\}$. Wählen wir insbesondere $D(a)$ aus $\mathfrak{U}_{n}$, dann gilt $u_{a, b}^{n}=1$. Solches Faktorensystem nennen wir normal; das wird aber nicht eindeutig durch 2 bestimmt.

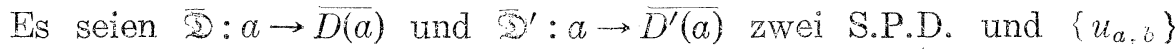
bzw. $\left\{u_{a, b}^{\prime}\right\}$ die zugehörigen Klassen der assoziierten Faktorensysteme. Ordnen wir dann $\overline{D(a) \times D^{\prime}(a)}$ (wo $\times$ das Kroneckersche Produkt bedeutet) dem $a$ zu, erhalten wir eine S.P.D. $\times \overline{1}^{\prime}: a \rightarrow D(a) \times D^{\prime}(a)$, der die Klasse der Faktorensysteme $\left\{u_{a, b} \cdot u_{a, b}^{i}\right\}$ zugeordnet wird. Die stetige

(10) Diese Definition von S.P.D. ist etwas verschieden von der von H. Weyl. Weyl fordert statt (3) die stärkere Bedingung, dass für passend gewählten Repräsentanten $D(a)$ aus der Klasse $\overline{D(a)}$ die Zuordnung von $\mathscr{G} \ni a \rightarrow D(\alpha) \in \Re_{n}$ eine stetige Abbildung von 3 in $\Omega_{n}$ sein soll. 
ganze Darstellung (S.G.D.) hat das Faktorensystem 1 und die S.P.D. $a \rightarrow \overline{D(a)^{*-1}}$ hat $\left\{u_{a, b}^{-1}\right\}$ als Faktorensystem, also bildet die Gesamtheit aller Klassen assoziierter Faktorensysteme verschiedener S.P.D. von (\$) nach der Multiplikationsregel $\left\{u_{a, b}\right\} \cdot\left\{u_{a, b}^{\prime}\right\}=\left\{u_{a, b} \cdot u_{a, b}^{\prime}\right\}$ eine Gruppe. Diese Gruppe heisst nach I. Schur( ${ }^{11}$ ) der Multiplikator von (G).

Wir können S.P.D. von (5) folgenderweise konstruieren. Es sei (3) eine topologische U̇berlagerungsgruppe von 13 ; es gelte nämlich

$$
\overline{(5)} / \overline{3} \cong(5)
$$

mit einem diskreten Normalteiler $\overline{4}$ aus dem Zentrum von (G) Es sei $D_{0}(\bar{a}), \bar{a} \in \overline{\mathbb{S}}$ eine stetige ganze Darstellung von $\overline{\mathscr{G}}$, bei der den Elementen

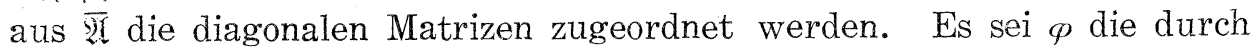
$\overline{(G)} / \bar{H} \cong$ (S) bestimmte stetige, homomorphe Zuordnung von $\overline{\mathbb{G}}$ auf (S), $(\phi(\bar{a})=a \in(\mathfrak{S})$, dann ist

$$
a \rightarrow \overline{D(a)}=\overline{D(\phi(\bar{a}))}=\overline{D_{0}(\bar{a})}
$$

eine S.P.D. von (53. Diese S.P.D. wollen wir die durch die S.G.D. $\mathfrak{D}_{0}: \bar{a} \rightarrow D_{0}(\bar{a})$ von $\overline{\mathscr{S}}$ induzierte S.P.D. von SS nennen.

Für die Frage, ob umgekehrt jede S.P.D. von (\$) durch eine S.G.D. von einer Überlagerungsgruppe $\bar{G}$ von $(\$)$ induziert wird, schicken wir den folgenden Hilfssatz voraus.

Hilfssatz. Es sei $\bar{D}: a \rightarrow D(a)$ eine beliebige S.P.D. von einer topologischen Gruppe (S). Für jedes Element a aus der genügend kleinen Umgebung t der Eins von \$ können wir Repräsentanten $D(a) \in \mathfrak{H}_{n}$ aus der Klasse $\overline{D(a)}$ so Wählen, dass die Zuordnung it $\ni a \rightarrow D(a) \in \mathfrak{U}_{n}$ eine homomorphe, stetige Zuordnung von it in $\mathfrak{1}_{n}$ ist.

Beweis. Es sei $0<\varepsilon<\frac{2 n}{4 n+3} \sin \frac{\pi}{2 n}$. Eine Umgebung 4 der Eins von (5) mit $t^{t}=t^{-1}$ sei so gewählt, dass das Bild von 1 durch S.P.D. (5) in der $\varepsilon$-Umgebung der Eins von $\mathfrak{P}_{n}$ enthalten ist. Wählen wir $D(a)$, $a \in \|$ aus der Klasse $\overline{D(a)}$ so, dass

$$
\rho_{0}\left(D(a), E_{n}\right)=\rho_{1}\left(\widehat{D(a)}, \tilde{E}_{n}\right)
$$

gilt, dann ist für $a, b$ aus it

$$
\begin{aligned}
\rho_{0}(D(a), D(b)) \leqq & \rho_{0}\left(D(a), E_{n}\right)+\rho_{0}\left(D(B), E_{n}\right) \\
& =\rho_{1}\left(\widetilde{D(a)}, \widetilde{E}_{n}\right)+\rho_{1}\left(\widehat{D(b)}, \widetilde{E}_{n}\right)<2 \varepsilon
\end{aligned}
$$

und, da für $\omega_{n}=e^{\frac{2 \pi i}{n}}$ und $i \neq 0(n)$

(11) Vgl. a.a.O. (5). 


$$
\rho_{0}\left(\omega_{n}^{i} E_{n}, E_{n}\right) \geqq \rho_{0}\left(\omega E_{n}, E_{n}\right)=2 \sqrt{n} \sin \frac{\pi}{2 n}
$$

ist, gilt, ferner

$$
\begin{aligned}
\rho_{0}\left(D(\alpha), D(b) \omega_{n}^{i}\right) & \geqq \rho_{0}\left(E_{n}, \omega_{n}^{i} E_{n}\right)-\rho_{0}\left(D(\alpha), E_{n}\right)-\rho_{0}\left(\omega_{n}^{i} D(b), \omega_{n}^{i} E_{n}\right) \\
& \geqq 2 \sqrt{n} \sin \frac{\pi}{2 n}-2 \varepsilon>2 \varepsilon \quad(i \neq 0(n))
\end{aligned}
$$

woraus folgt $\rho_{0}(D(a), D(b))=\rho_{1}(\widehat{D(a)}, \widehat{D(b)}), a, b \in 1 \mathrm{t}$. Daher ist die Zuordnung $a \rightarrow D(a)$ von it in $\Omega$ eine stetige.

Aus $D(a) \cdot D(b)=u_{a, b} D(a b), u_{a, b}^{n}=1$ folgt andererseits für $a, b, a b \in \downarrow$

$$
\begin{aligned}
& \rho_{0}\left(u_{a, b} E_{n}, E_{n}\right)=\rho_{0}\left(E_{n}, D(a) D(b) D(a b)^{-1}\right) \\
& \quad \leqq \rho_{0}\left(E_{n}, D(a)\right)+\rho_{0}(D(a), D(a) D(b))+\rho_{0}\left(D(a) D(b), D(a) D(b) D(a b)^{-1}\right) \\
& \quad \leqq \rho_{0}\left(E_{n}, D(a)\right)+\|D(a)\| \rho_{0}\left(E_{n}, D(b)\right)+\|D(a)\| \cdot\|D(b)\| \cdot \rho_{0}\left(E_{n}, D(a b)^{-1}\right) \\
& \quad \leqq=(\sqrt{n}+\varepsilon) \varepsilon+(\sqrt{n}+\varepsilon)^{2} \varepsilon \leqq(4 n+3) \varepsilon<2 n \sin \frac{\pi}{2 n},
\end{aligned}
$$

weil $D(a b)^{-1}=D\left((a b)^{-1}\right)$ und $\|D(a)\| \leqq\left\|E_{n}\right\|+\rho_{0}\left(E_{n}, D(a)\right)$ ist. Also folgt $\left|1 \mathrm{t}_{a, b}-1\right|<2 \sqrt{n} \sin \frac{\pi}{2 n}$ oder $u_{a b}=1$, daher ist die Zuordnung $a \rightarrow D(a)$ von $1 t$ in $\Re_{n}$ sogar eine homomorphe.

Für die O-z.h. topologische Gruppe $\$$ folgt sofort aus diesem Hilfssatz der folgende

Satz 10. Jede S.P.D. (1) der O-zusammenhängenden topologischen Gruppe (\$) ist durch eine S.G.D. $\mathfrak{D}_{0}$ einer Wegeklassengruppe $\mathfrak{S}_{\mathfrak{G}}(\mathfrak{b})$ für genügend kleine Umgebung v der Eins von (S) induziert.

Beweis. Eine Umgebung it der Eins von (9) sei so klein gewählt, dass für sie der Hilfssatz gilt, und $\mathfrak{b}$ eine Umgebung der Eins von (f) mit $\mathfrak{v}^{2}<\mathfrak{t}$. Dem $\mathfrak{b}$-Wege

$$
W(e, a)=\left\{e, a_{1}, a_{1} a_{2}, \ldots, a_{1} \ldots a_{r^{*}}=a\right\} \text { mit } a_{i} \in \mathfrak{v}
$$

ordnen wir die Matrix

$$
D_{0}(W(e, a))=D\left(a_{1}\right) \cdot D\left(a_{2}\right) \ldots D\left(a_{r}\right) \in \mathfrak{U}_{n},
$$

zu, dann ist $D_{0}(W(e, a))=D_{0}\left(W^{\prime}(e, a)\right)$, falls $W(e, a) \sim W^{\prime}(e, a)$ (b) ist, denn es gilt ja dabei $W(e, a) \equiv W^{\prime}(e, a)(1 t)$ und $a \rightarrow D(a)$ ist eine homomorphe Zuordnung von it in $\AA_{n}$. Diese Zuordnung $W(e, a) \rightarrow D_{0}(W(e, a))$ ist also eine S.G.D. $\mathfrak{D}_{0}$ von $\mathfrak{S}_{\mathfrak{B}}(\mathfrak{b})$, und es gilt

$$
\left.\overline{D_{0}(W(e, a)}\right)=\overline{D(a)}, a \in(\mathfrak{s} \text {. }
$$


Korollar. D $: s \rightarrow D(s)$ sei eine S.P.D. von einer O-zusammenhängenden, im Kleinen O-zusammenhängenden und O-einfach-zusammenhängenden Gruppe. Dann ist der Multiplikator $M=1$, und für passend gewählte $D(s)$ aus $D(s)$ ist $D: s \rightarrow D(s)$ eine stetige ganze Darstellung.

Denn in diesem Falle ist in der obigen Betrachtung $\$ 1 \mathfrak{S}_{\mathfrak{G}}(\mathfrak{b})=\mathbb{G}$, also $\overline{\mathscr{S}}=\left(\mathbb{S}\right.$ ) und $\mathfrak{S}_{0}=\mathfrak{D}$.

Es gilt aber im allgemeinen nicht notwendig O-zusammenhängenden topologischen Gruppen der folgende

Satz 11. Jede S.P.D. $\bar{D}$ vom Grade $n$ der topologischen Gruppe (3) ist durch eine S.G.D. $\mathfrak{S}_{0}$ einer höchstens n-blättrigen Überlagerungsgruppe (S) von 19 induziert.

Falls (5) O-zusammenhängend und im Kleinen O-zusammenhängend ist, können wir $\bar{G}$ so wählen, dass $\bar{G}$ auch O-zusammenhängend und im Kleinen O-zusammenhängend ist.

Beweis. 1) Durch die gegebene S.P.D. 5 sei $\overline{D(a)} \in \mathfrak{P}_{n}$ dem $a \in(\$)$ zugeordnet. Es sei $D(a)$ ein aus $\mathfrak{H}_{n}$ gewählten Repräsentant von $\widehat{D(a)}$, und $u_{a, b}=\omega_{n}^{c(a, b)}, \omega_{n}=e^{\frac{2 \pi i}{n}}(\sigma(a, b)=0,1, \ldots, n-1)$ dazu gehöriges Faktorensystem. Jedem Element $s$ aus (5) entsprechend führen wir ein abstraktes Element $U_{s}$ ein. $A$ sei ein weiteres abstraktes Element. Dann bilden wir eine Gruppe $\mathbb{S}_{0}$ mit dem Erzeugendensystem $\left\{U_{s},(s \in(\mathbb{S}) ; A\}\right.$ und mit den definierenden Relationen:

$$
\begin{aligned}
A^{n} & =1, \\
A U_{s} & =U_{s} A, \\
U_{s} U_{t} & =A^{\sigma(s, t)} U_{s t} .
\end{aligned}
$$

Jedes Element von $\mathfrak{G}_{0}$ lässt sich dann eindeutig in $\operatorname{der}$ Form $U_{s} A^{\sigma}$ darstellen, und die Untergruppe $\mathfrak{U}=\{A\}$ liegt im Zentrum von $\mathfrak{G}_{0}$.

Nun topologisieren wir $\mathbb{G}_{0}$ fologendermassen: für eine Umgebung it der Eins von $(S)$ und eine Umgebung $\mathfrak{H}^{0}$ von $E_{n}$ von $\mathfrak{U}_{n}$ definieren wir die $\mathfrak{U}\left(\mathfrak{u}, \mathfrak{u}^{0}\right)$-Umgebung von $U_{s} A^{\sigma} \in \mathbb{G}_{0}$ als Gesamtheit aller Elemente $U_{t} A^{\tau}$ mit folgenden Eigenschaften:

$$
\begin{aligned}
& t \in \mathcal{S} \cdot \mathfrak{u} \text { in (B), } \\
& D(t) \omega_{n}^{\bar{v}} \in D(s) \omega_{n}^{\sigma} \mathfrak{U}^{0} \text { in } \mathfrak{U}_{n} .
\end{aligned}
$$

Da (G) und $\mathfrak{U}_{n}$ topologische Gruppen, und da $D(s) D(t)=\omega_{n}^{\sigma(s, t)} D(s t)$ ist, folgt dass $\bar{G}_{0}$ auch eine topologische Gruppe ist.

Es sei $\phi$ eine Zuordnung von $\overline{\mathscr{G}}_{0}$ auf $(5)$ definiert durch $\phi\left(U_{s} A_{\sigma}\right)=s$. Nach der Relation 3) ist $\varphi$ ersichtlich eine homomorphe Zuordnung. Es 
ist ausserdem eine stetige und gebietstreue Zuordnung. Denn das $\varphi^{-}$ Urbild einer Umgebung $\mathfrak{u}$ der Eins von $(3)$ ist nach der Definition von $\phi$ eine Umgebung $\mathfrak{U}\left(\mathfrak{u}, \mathfrak{U}_{n}\right)$ der Eins in $\bar{G}_{0}, \varphi$ ist also stetig. Um nachzuweisen, dass $\phi$ gebietstreu ist, geuügt zu zeigen, dass das $\phi$-Bild von $\mathfrak{U}\left(\mathfrak{t}, \mathfrak{H}^{0}\right)$-Umgebung von $U_{s} A^{\sigma}$ eine offene Menge in (S) ist. Dazu hat man nach der Definition von $\mathbb{H}\left(\mathfrak{H}, \mathfrak{H}^{0}\right)$-Umgebung nur zu zeigen, dass die Gesamtheit aller Elemente $t$, so dass $1^{\circ}\left(D(s) \omega_{n}^{\sigma}\right) \ni D(t) \omega_{n}^{p}$ mit einem passenden $\tau$ gilt, eine offene Menge in $\left(3\right.$ bildet. Wegen $\mathbb{U}_{n} / \$_{n}^{0}=\Re_{n}$ wird nun $\mathfrak{U}_{n}$ auf $\mathfrak{P}_{n}$ homomorph abgebildet. Es sei $f$ diese Abbildung und $f\left(\mathfrak{H}^{0}\right)=\widetilde{\mathfrak{H}}^{0}$. Dann bedeutet $\mathfrak{H}^{0}\left(D(s) \omega_{n}^{\sigma}\right) \ni D(t) \omega_{n}^{\tau}$ mit einem passenden $\tau$ dasselbe wie $\widetilde{\mathfrak{H}}^{0} \ni \widehat{D(t)}$. Da aber die Zuordnung $\& \ni t \rightarrow \widetilde{D(t)} \in \Re_{n}$ nach der Definition von S.P.D. (5) stetig ist, bildet die Gesamtheit von $t$ mit $\widetilde{D(t)} \in \tilde{\mathbb{1}} 0$ eine ofiene Menge in $(\$)$ Daher ist $\phi$ gebietstreu.

Nun ist die Untergruppe $\mathfrak{U}=\{A\}$ gerade die Gesamtheit aller Elemente aus $\overline{\mathscr{G}}_{0}$, die durch $\varphi$ auf 1 abgebildet werden: Es gilt also

$$
\overline{\mathrm{G}}_{0} / \mathfrak{U} \cong(\mathfrak{S},
$$

und $\overline{\mathrm{S}}_{0}$ und $(\$)$ sind im Kleinen topologisch isomorph. Wir sehen ferner sofort, dass die S.G.D. $\mathfrak{D}_{0}^{*}: U^{s} A^{\sigma} \rightarrow D(s) \omega_{n}^{\sigma}$ von $\bar{G}_{0}$ die S.P.D. $\bar{D}$ von (S) induziert.

Es sei nun (3 O-z.h. und im Kleinen O-z.h. Da die eben konstruierte topologische Gruppe $\overline{\mathbb{G}}_{0}$ mit (3) im Kleinen topologisch isomorph ist, ist also $\overline{\mathbb{S}}_{0}$ im Kleinen O-z.h., aber nicht immer O-z.h. Also sei $\overline{\mathbb{S}}_{1}$ die OKomponente von Eins in $\overline{\mathbb{G}}_{0}$, d.h. die grösste die Eins enthaltende Ozussmmenhängende Untermenge von $\overline{G_{0}}$. Es lässt sich wie üblich beweisen, dass $\overline{G_{1}}$ ein abgeschlossener Normalteiler von $\overline{G_{3}}$ ist. Da $\bar{G}_{0}$ im Kleinen O-z.h. ist, enthält $\overline{\mathscr{S}}_{1}$ eine Umgebung der Eins von $\overline{\mathscr{S}}_{0}$. Daraus folgt sofort $\varphi\left(\overline{\mathscr{S}}_{1}\right)=\left(\mathbb{S}\right.$, da $\left(\mathbb{S} \mathrm{O}-\mathrm{z} . \mathrm{h}\right.$. ist. Also gilt für $\mathfrak{A}_{1}=\mathfrak{A} \cap \overline{\mathrm{G}}_{1}$

$$
\overline{\mathbb{G}}_{1} / \mathfrak{A}_{1} \cong \mathbb{Z}
$$

und $\overline{\mathscr{B}}_{1}$ ist wieder eine Überlagerungsgruppe von $\$$, deren Existenz im Satz behauptet wurde. Wir sehen leicht ein, dass $\overline{\mathcal{G}}_{1}$ von der Wahl von $D(s)$ aus $U_{n}$ unabhängig durch $\$$ allein eindeutig bestimmt ist.

2) Falls (5) O-z.h. und im Kleinen O-z.h. ist, können wir $\bar{G}_{1}$ aus der Wegeklassengruppe $\mathfrak{B}_{\mathfrak{s}}(\mathfrak{b})$ im Satz 10 konstruieren. $D_{0}(W(e, a)) \in U_{n}$ sei die im Satz 10 konstruierte S.G.D. von $\mathfrak{S}_{\mathfrak{s}}(\mathfrak{b})$. Dann ist $D_{0}(W(e, e))=\omega_{n}^{i} E_{n}$ aus (23). Die Gesamtheit $\mathfrak{\mho}_{0}$ aller $\mathfrak{b}$-Wegeklassen aus $\mathfrak{F}_{\mathfrak{s}}(\mathfrak{b})$ mit $D_{0}(W(e, e))=E_{n}$ bildet nun einen diskreten Normalteiler von $2 \mathfrak{S}_{\mathfrak{G}}(\mathfrak{b})$ mit $\left(\mathfrak{F}_{\mathfrak{S}}(\mathfrak{v}): \widetilde{F}_{0}\right) \leqq n$. Die Faktorgruppe $\mathfrak{S}_{\mathfrak{S}}(\mathfrak{b}) / \widetilde{\mho}_{0}=\overline{\mathbb{S}}$ ist also eine O-zusammenhängende und im Kleinen O-zusammenhängende endlichvielblättrige 
Überlagerungsgruppe von (S) . Da $D_{0}(W)=E_{n}$ für $W \in \mathfrak{r}_{0}$ ist, lässt sich $\mathfrak{D}_{0}$ als die S.G.D. von $\bar{G}$ ansehen, welche S.P.D. (5) von (S) induziert.

Nunmehr zeigen wir, dass die Gruppen $\overline{\mathbb{S}}_{1}$ und $\overline{\mathbb{S}}$, die in 1) bzw. 2) konstruiert werden, topologisch isomorph sind, d.h. dass es durch eine topologisch isomorphe Zuordnung $f$ von $\overline{\mathscr{G}}$ auf $\overline{\mathscr{G}}_{1}: \overline{\mathbb{G}} \ni T^{\prime} \rightarrow f\left(T^{\prime}\right)=T \in \overline{\mathscr{G}}_{1}$ gibt, und zwar dabei $D_{0}\left(T^{\prime}\right)=D_{0}^{*}(T)$ gilt. Es sei $\psi$ die stetige homomorphe Zuordnung von $\overline{\mathbb{S}}=\mathfrak{S}_{\mathfrak{S}}(\mathfrak{b}) / \mathfrak{F}_{0}$ in 2) auf $\left(\mathfrak{S}\right.$ und $U_{s}^{\prime}$ sei ein Element aus $\overline{G S}$ mit $\psi\left(U_{s}^{\prime}\right)=s \in G . \widetilde{\mho}_{\mathfrak{S}}(\mathfrak{b}) / \mathfrak{F}_{0}$ ist eine zyklische Grupph von der Ordnung $m(m=$ ein Teiler von $n) ; A^{\prime}$ sei ein erzeugendes Element der Faktorgruppe $\mathfrak{U}^{\prime}=F_{\mathbb{S}}(\mathfrak{b}) / \widetilde{\mho}_{0}$. Dann kann man (S) als eine Gruppe ansehen, die aus (5) nach Erweiterung mit $\mathfrak{H}^{\prime}$ entsteht, und die Struktur von $\overline{(S)}$ ist durch die Relationen

$$
U_{s}^{\prime} A^{\prime}=A^{\prime} U_{s}^{\prime}, \mathrm{A}^{\prime m}=1 \quad \text { und } \quad U_{s}^{\prime} U_{t}^{\prime}=A^{\prime \sigma^{\prime}(s, t)} U_{s t}^{\prime}
$$

eindeutig bestimmt. Da nach der Definition von $\mathfrak{D}_{0}$ in 2) $D_{0}\left(U_{s}^{\prime}\right)=D(s)$ $(D(s)$ ist ein Repräsentant aus der Klasse $\overline{D(s)} \in \mathfrak{D}), D\left(A^{\prime}\right)=\omega_{n}{ }^{\frac{n}{m}}$ ist, gilt

$$
D(s) D(t)=\omega_{n}^{\sigma(s, t)} D(s t),
$$

wobei wir $\sigma(s, t)=\frac{n}{m} \sigma^{\prime}(s, t)$ setzen. Konstruieren wir also ${\overline{G_{0}}}_{0}$ in 1$)$ mit diesen $\sigma(s, t)$ und $D(s)$, so ist $\overline{(S)}$ durch die Zuordnung $f: U_{s}^{\prime} \rightarrow U_{s}$, $A^{\prime} \rightarrow A^{\frac{n}{m}}$ isomorph in $\overline{\mathscr{G}}_{0}$ abgebildet. Gilt für $k \rightarrow \infty U_{t_{k}}^{\prime} A^{\prime} \tau_{k}=T_{k}^{\prime} \rightarrow T^{\prime}=U_{t}^{\prime} A^{\circ}$ in $\overline{B S}$, dann gilt auch $t_{k}=\psi\left(T_{k}^{\prime}\right) \rightarrow t=\psi\left(T^{\prime}\right)$ und $D_{0}\left(T_{k}^{\prime}\right) \rightarrow D_{0}\left(T^{\prime}\right)$, da $\psi$ und $D_{0}$ stetige Abbildungen sind. Also gilt $T_{k}=f\left(T_{k}^{\prime}\right) \rightarrow T=f\left(T^{\prime}\right)$ in $\bar{G}_{0}$. Sei nun umgekehrt $f\left(T_{k}^{\prime}\right) \rightarrow f\left(T^{\prime}\right)$ in $\bar{G}_{0} . \quad \psi^{-1}$ ist eine endlichvielwertige Zuordnung von (S) auf $\bar{G}$. Aus $t_{k} \rightarrow t$ folgt also $U_{t_{k}}^{\prime} A^{\prime p_{k}} \rightarrow U_{t}^{\prime} A^{\prime \tau}$ mit passendem $\rho_{k}$ nach der im Kleinen topologischen Isomorphie von $\mathbb{G}$ und (G). Andererseits folgt $D_{0}^{*}\left(f\left(T_{k}^{\prime}\right)\right)=D\left(t_{k}\right) \omega_{n}^{\frac{n}{m} \tau_{k}} \rightarrow D_{0}^{*}\left(f\left(T^{\prime}\right)=D(t) \omega_{n}^{\frac{n}{m} \tau}\right.$ aus $f\left(T_{k}^{\prime}\right) \rightarrow f\left(T^{\prime}\right)$, und $D_{0}\left(U_{t_{k}}^{\prime} A^{\prime \rho k}\right)=D\left(t_{k}\right) \omega_{n}^{\frac{n}{m} k} \rightarrow D_{0}\left(U_{t} A^{\prime \tau}\right)=D(t) \omega_{n}^{\frac{n}{m}}$ aus $U_{t_{k}}^{\prime} A^{\prime \rho} \rho_{k \rightarrow} U_{t}^{\prime} A^{\prime \tau}$ in $\overline{(S)}$. Also muss $\rho_{k}=\tau_{k}$ für $k>N$ sein, d.h. $T_{n}^{\prime} \rightarrow T$ in $\bar{S}$. Daher ist die Zuordnung $f$ eine topologische isomorphe von $\overline{\mathscr{S}}$ in $\overline{\mathbb{G}}_{0}$. Da ferner $\overline{(S)}$ O-z.h. ist, muss $f(\overline{\mathbb{G}})$ auch O-z.h. sein, also $\overline{\mathbb{G}_{1}}>f(\overline{\mathbb{G}})$ nach der Definition von $\overline{\mathbb{S}}_{1}$. Andererseits sind $\overline{\mathbb{B S}}_{1}$ und $\overline{\mathbb{S}}$ (order $f(\overline{\mathrm{G}})$ ) im Klelnen topologisch isomorph; $f(\$)$ enthält also eine Umgebung von Eins von $\bar{G}_{1}$. Nach dem ersten Fundamentalsatz von Schreier (S. 1) ist also $\overline{\mathbb{G}}_{1}=f(\overline{\mathrm{G}})$, w.z.b.w.

2. Von nun an betrachten wir ausschliesstich kompakte metrische Gruppen. 
Satz. 12. Es sei (S) eine kompakte, metrische topologische Gruppe. Dann ist jede S.P.D. vollständig reduzibel.

Beweis. Aus der Kompaktheit von $\mathbb{S}$ folgt die Kompaktheit von $\overline{\mathbb{G}}_{0}$ im Satz 11, da $\overline{\mathbb{S}}_{0}$ eine endlichvielblättrige Úberlagerungsgruppe von (S) ist. Also gibt es eine unitär unimodulare Darstellung $\mathfrak{D}_{1}^{\prime}$ von $\overline{\mathbb{G}}_{0}$, welche mit $\mathfrak{D}_{0}^{*}$ im Satz 11 äquivalent ist. Da $\mathfrak{D}_{0}^{\prime}$ eine mit $\mathfrak{S}$ äquivalente S.P.D. von (S) induziert, folgt die vollständige Reduzibilität von $\bar{S}$ üblicherweise.

Satz 13. Es sei (3) eine komparkte, metrische zusammenhängende, im Kleinen zusammenhängende. topologische Gruppe. Dann könneu alle S.P.D. von (G), deren Faktorensysteme zu einem gegebenen Faktorensystem assoziiert sind, von den S.G.D. einer bestimmten endlichen Überlagerungs-

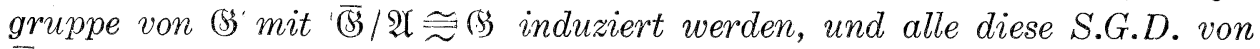
$\overline{(3)}$ geben sämtlich die gleiche Darstellung von $\mathfrak{U}$.

Beweis. Zuerst zeigen wir, dass die Gruppe $\overline{(S}_{1}$ in 1) im Beweis des Satzes 11, die der S.P.D. $\bar{D}$ entspricht, mit der Gruppe $\overline{\mathbb{G}_{1}^{\prime}}$ topologisch isomorph ist, die statt $\overline{\mathrm{D}}$ der S.P.D.

$$
\overline{\mathfrak{S}}^{\prime}=(\stackrel{\mathfrak{D}}{\cdot} \cdot \underbrace{m}_{\mathfrak{D}})
$$

entspricht. Sei nämlich $\overline{\mathscr{G}}_{0}^{\prime}$ die der Gruppe $\overline{\mathscr{G}}_{0}$ entsprechende Gruppe für $\bar{D}^{\prime}$, üann ist $\bar{G}_{0}$ ersichtlich in $\overline{\mathscr{G}}_{0}^{\prime}$ mit dem Index $m$ stetig isomorph eingebettet. Da aber $\overline{\mathscr{G}}_{0}$ und $\overline{\mathbb{S}}_{0}^{\prime}$ beide im Kleinen topologisch mit $\mathbb{S}^{3}$ sind, stimmen die Eins enthaltende O-Komponente von $\overline{\mathbb{G}}$ und $\overline{\mathbb{S}_{0}^{\prime}}$ nach dem 1. Fundamentalsatz von Schreier überein. Es ist dabei klar, dass die S.G.D. $\mathfrak{D}_{0}$ und $\mathfrak{D}_{0}^{\prime}$ von $\bar{G}_{1}$ bzw. $\overline{\mathscr{G}}_{1}^{\prime}$, welche $\bar{S}$ bzw. $\mathbb{S}^{\prime}$ von $(S)$ induzieren, die gleiche Darstellung von $\mathfrak{A}$ bzw. $\mathfrak{U}^{\prime}$ geben, wobei $\bar{\Theta}_{1} / \mathfrak{A} \cong\left(\mathbb{S}, \overline{\mathscr{S}}^{\prime} / \mathfrak{A Y}^{\prime} \cong(\mathfrak{S}\right.$ sind.

Es seien nun 5 , $\mathfrak{D}^{\prime}$ zwei beliebige S.P.D. von den Graden $n$ bzw. $n^{\prime}$, deren Faktorensysteme zueinander assoziiert sind. Dann bilden wir die S.P.D. $n n^{\prime}$-ten Grades

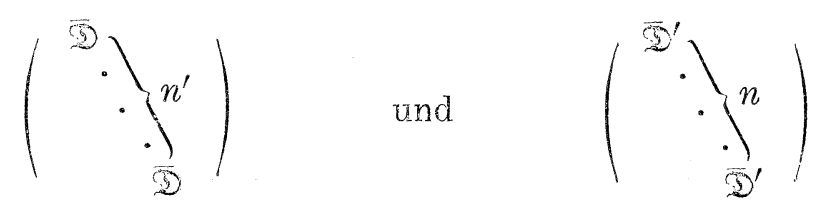

und erhalten wieder S.P.D. mit zueinander assoziierten Faktorensystemen. Nach dem eben gezeigten genügt es also den Satz nur für den Fall von zwei S.P.D. mit gleichem Grade $m$ zu beweisen. 
Wählen wir nun die Repräsenten $D(s)$ bzw. $D(s)^{\prime}$ aus der Klasse $\overline{D(s)}$ von $\overline{5 z w} \overline{D(s)^{\prime}}$ von $\bar{\Phi}^{\prime}$ so, dass sie gleiche Faktorensysteme haben. $\overline{\mathcal{G}}_{0}$ sei die abstrakte Gruppe mit den Erzeugenden $\left\{U_{s} ; A\right\}$ und mit den Relationen 1) 2) 3) wie in 1) im Beweis des Satzes 11. Wir topologisieren dann $\bar{G}_{0}$, indem wir die Gesamtheit aller Elemente $U_{t} A^{\tau}$ mit

$$
\begin{aligned}
& s \mathfrak{t} \ni t \text { in } \quad \mathfrak{G}, \\
& \mathfrak{U}^{0}\left(D(s) \omega_{m}^{\sigma}\right) \ni D(t) \omega_{m}^{\tau} \text { in } \mathfrak{U}_{m}, \\
& U^{T^{0}}\left(D^{\prime}(s) \omega_{m}^{\sigma}\right) \ni D(t) \omega_{m}^{\circ} \text { in } U_{m},
\end{aligned}
$$

als $\mathfrak{H}\left(\mathfrak{u}, \mathfrak{H}^{0}, \mathfrak{H}^{\prime}\right)$-Umgebung von $U_{s} A^{\sigma}$ definieren. Dann zeigt sich wie bei Satz 11. 12, dass $\overline{\mathcal{G}}_{0}$ eine kompakte metrische topologische Gruppe ist. Die abstrakte Gruppe $\overline{G_{0}}$ lässt sich noch auf zweierlei Weise topologisieren: erstens kann man nämlich die Gesamtheit aller $U_{t} A^{\tau}$, die den Bedingungen 1), 2) genügen, als $\mathfrak{H}\left(\mathfrak{t}, \mathfrak{U}^{0}\right)$-Umgebung definieren ; zweitens die Gesmmtheit der $U_{t} A^{\tau}$, die 1), 3) genügen, als $\mathbb{H}\left(\mathfrak{t t}, \mathfrak{H}^{\prime \prime}\right)$-Umgebung. Die mit diesen Umgebungssystemen topologisierten Gruppen seien bzw. $\overline{\mathscr{G}}_{0}^{*}$ und $\overline{\mathbb{G}}_{0}^{* \prime}$, und $\overline{\mathscr{G}}_{1}^{*}$, $\overline{\mathscr{G}}_{1}^{* \prime}$ seien bzw. die O-Komponente von $\overline{\mathscr{S}}_{0}^{*}, \overline{\mathscr{G}}_{0}^{* \prime}$. Dann ist die identische Zuordnung von $\overline{(5)}$ auf sich eine stetige Zuordnung von $\overline{\mathbb{G}}_{0}$ auf $\overline{\mathbb{G}}_{0}^{*} \mathrm{bzw}$. $\overline{\mathcal{G}}_{0}^{* \prime}$. Sie ist sogar eine homöomorphe Zuordnung, da $\overline{\mathbb{G}_{0}}$ kompakt metrisch ist. Also müssen die O-Komponenten $\overline{\mathbb{G}}_{1}^{*}$ und (5).' topologisch isomorph sein. Der letzte Teil des Satzes ist ohne weiteres klar.

Korollar. Wenn eine S.P.D. (D) $s \rightarrow \overline{D(s)}$ einer kompakten metrischen zusammenhängenden, im Kleinen zusammenhängenden Gruppe ein mit 1 assoziiertes Faktorensystem besitzt, dann können wir Repräsentanten $D(s)$ aus der Klasse so wählen, dass $s \rightarrow D(s)$ eine S.G.D. ist.

Denn im Beweis des Satzes 12 können wir also $\aleph^{\prime}$ eine S.G.D. wählen, dann ist $\bar{G}_{1}=$ (5) nach Satz 13, die Behaupting ergibt sich also nach Satz 11.

Nach Satz 12 reduziert sich die Untersuchung der S,P.D. auf der irreduziblen. Dafür gilt

Satz 14. Es sei (D) eine S.P.D. mit dem Faktorensystem $u_{\alpha, b}$ einer kompakten metrischen zusammenhängenden, im Kleinen zusamenhängenden topologischen Gruppe (3) . Dann ist jede S.P.D. S' $^{\prime}$ it einem zu $u_{a, b}$ assoziierten Faktirensystem in einem irreduziblen Bestandteil von $\mathfrak{D}_{0} \times \mathbb{D}$ enthalten, wobei $\mathfrak{D}_{0}$ eine passend gewählte S.G.D. zon (S) ist.

Beweis. Es sei $D(a)$ ein unitärer, unimodularer, Repräsentant der Klasse $\overline{D(a)}$, dann ist $\bar{D}^{*}: a \rightarrow \overline{D(a)^{*}}$ eine S.P.D mit dem Faktorensystem $u_{a, b}^{*}=u_{a, b}^{-1}$, wobei $*$ konjugiert-komplexe Zahl bedeutet. Nach dem 
Korollar von Satz 13 können wir daher Repräsentanten $D_{0}(a)$ aus den Klassen $\overline{D^{\prime}(a) \times D(a)^{*}}$ so wählen, dass $a \rightarrow D_{0}(a)$ eine S.G.D. ist.

Es sei nun $\bar{G}$ die im Satz 11 angegebene U̇berlagerungsgruppe von (S) fur die S.P.D. $\bar{S}$, und $\mathfrak{S}_{0}^{\prime}$ die S.P.D. von $\bar{S}$, welche $\overline{2}$ induziert. Dann enthält die S.G.D. $\mathfrak{S}_{0}^{\prime} \times \mathfrak{S}_{0}^{\prime *}$ von $\overline{\mathfrak{S}}$ unter ihren irreduziblen Bestandteilen sicher mindestens einmal die Hauptdarstellung von $\bar{G}$, da $\bar{G}$ kompakt metrisch ist $\left({ }^{12}\right)$. Also enthält auch $5 \times \Sigma^{*}$ mindestens einmal die Haupt. darstellung von 8 unter den irredrziblen Bestandteilen. Dann folgt die Behauptung aus

$$
\left.\overline{\mathfrak{D}} \times \mathfrak{D}_{0}=\overline{\mathfrak{D}} \times(\overline{\mathfrak{D}})^{\prime} \times \overline{\mathfrak{D}}^{*}\right)=\left(\overline{\mathfrak{D}} \times \overline{\mathfrak{D}}^{*}\right) \times \overline{\mathfrak{D}}^{\prime} .
$$

Über die Anzahl verschiedener Faktorensysteme, oder über die Struktur des Multiplikators $\mathfrak{M}$ gilt

Satz 15. Es sei (S) eine zusammenhängende, im Kleinen zusammenhängende und im Kleinen einfach-zusammenhängende kompakte, metrische topologische Gruppe. $\mathfrak{W}_{\mathfrak{S}}(0)^{\prime}$ sei die Kommutatorgruppe der O-Wegeklssengruppe $\mathfrak{B}_{\mathfrak{S}}(0), \widetilde{\mho}^{\prime}=\widetilde{\mho}_{\mathfrak{s}}(0) \cap \mathfrak{B}_{\mathrm{S}}(0)^{\prime}$, und $\chi^{(e)}\left(\widetilde{\mho}^{\prime}\right)$ die Gruppe der Charaktere endlicher Ordnungen von $\Im^{\prime}$. Dann gilt

$$
M\left((S) \cong \chi^{(e)}\left(\widetilde{F}^{\prime}\right)\right. \text {. }
$$

wobei M(B) der Multiplikator vou (\$3 bedeutet.

Beweis. Wegen $\mathfrak{S}_{\mathfrak{s}}(0) / \mathscr{F}_{\mathfrak{s}}(0) \cong\left(\mathbb{S}\right.$ wird $\mathfrak{W}_{\mathfrak{S}}(0)$ auf (5) homomorph zugeordnet. Es sei $U_{s} \in \mathfrak{S}_{\mathfrak{s}}(0)$ ein beliegiger Repräsentant von $\mathfrak{S}_{\mathfrak{s}}(0)$ mod. $\widetilde{\mho}_{s}(0)$, der bei dieser Zuordnung dem $s \in \mathbb{S}$ entspricht. Dann ist

$$
U_{s} U_{t}=A_{s, t} U_{s t} \text { mit } A_{s, t} \in \widetilde{F}_{\sigma}(0) \text {. }
$$

Es sei nun $\chi$ ein Charakter endlicher Ordnung von $\mho_{s}(0)$, und $\mho_{0}$ die Gesamthcit aller Eltmente $A$ von $\widetilde{\mho}_{\mathfrak{s}}(0)$ mit $\chi(A)=1$, dann ist $\left(\widetilde{\mho}_{\mathfrak{B}}(0): \mathscr{F}_{0}\right)=n$ : endlich, und $\mathfrak{B}_{\mathfrak{s}}(0) / \mathscr{F}_{0}=\overline{\mathbb{G}}$ eine kompakte metrische Gruppe. Nun zeigen wir, dass es mindestens eine irreduzible S.G.D. (D) von $\overline{G S}$ mit $D(A)=\chi(A) E_{m}\left(A \in \tilde{F}_{\mathfrak{s}}(0) / \widetilde{\mho}_{0}\right)$ gibt.

Dazu genügt es zu zeigen, dass die Gruppe $\left\{\chi^{\prime}\right\}$ der Charaktere $\chi^{\prime}$ von $\mathfrak{\mho}_{\mathfrak{s}}(0) / \widetilde{\mho}_{0}$, für welche $D(A)=\chi^{\prime}(A) \cdot E$ für eine gewisse irreduzible

(12) $\chi$ sei der zu der Darstellung $\mathscr{D}_{0}^{\prime}$ gehörige Charakter von $\bar{G}$, und $\chi_{0}$ der Hautpcharakter. Es sei

$$
x \bar{x}=x_{1}+\cdots+x_{r}
$$

die Zerlegung von $\bar{x} \bar{x}$ in die Summe der einfachen Charaktere $\chi_{i}$ von $\overline{\text { F }}$. Dann gilt

$0 \neq \int \chi \bar{x} d x=\int \chi_{1} \bar{\chi}_{0} d x+\cdots+\int \chi_{r} \bar{\chi}_{0} d x=$ Anzahl der Hauptcharaktere in $\left\{\chi_{i}\right\}$, also gibt es mindestens einmal die Hauptdarstellung unter den irreduziblen Bestandteilen von der Darstellung $\mathscr{D}_{0} \times \mathscr{D}_{0} *$ von $\overline{\mathscr{O}}$. 
S.G.D. D von (S) gilt, mit $\chi^{(e)}\left(\widetilde{\mho}_{\mathscr{G}}(0) / \widetilde{\mho}_{0}\right)$ übereinstimmen. Wäre also $\left\{\chi^{\prime}\right\}$

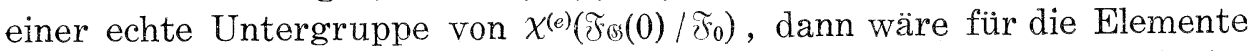
$A$ von $\mathfrak{F}_{\mathfrak{G}}(0) / \mathfrak{F}_{0}$ mit $\chi^{\prime}(A)=1$ für alle $\chi^{\prime} \in\left\{\chi^{\prime}\right\}$. Da aber $\mathfrak{F}_{\mathfrak{G}}(0) / \mathfrak{F}_{0}$ im Zentrum von $\overline{\mathbb{G}}$ enthalten ist, wäre auch $D(A)=E$ für alle irreduziblen S.G.D. von (S) Also muss solches Element nur die Eins von $\overline{(5)} \operatorname{sein}\left({ }^{13}\right)$, was ein Widersprspruch ist.

Der von einer S.G.D. D von $\overline{(S)}$ induzierierten S.P.D. $\bar{D}$ von $(\mathbb{S}$ wird das Faktorensystem $u_{a, b}=\chi\left(A_{a, b}\right)$ zugeordnet, wobei $A_{a, b} \in F_{\mathfrak{s}}(0)$ durch (24) bestimmt sind. Also wird jedem Charakter endlicher Ordnung von $F_{\mathscr{S}}(0)$ eindeutig ein Element des Multiplikators M(S) zugeordnet; diese Zuordnung ist eine homomorphe Zuordnung von $\chi^{(e)}\left(\mathscr{F}_{\mathfrak{S}}(0)\right)$ in $\mathfrak{M}(\mathbb{S})$. Dabei werden sogar alle Elemente von $\mathfrak{M}_{(\$ S)}$ betroffen, da nach Satz 10 , 11, jede S.P.D. von (S) von einer S.G.D. von $\mathfrak{S}_{\mathfrak{S}}(0)$ induziert wird, welche einem endlichen Charakter von $\mathfrak{F}_{\mathfrak{B}}(0)$ entsprechen.

Es sei nun $\chi$ ein Charakter endlicher Ordnung von $\mathfrak{F}_{\mathfrak{s}}(0)$, der bei der ebengenannten Zuordnung der Eins von $\mathfrak{M}$ zugeordnet wird. Dann gilt $\chi\left(A_{s, t}\right)=\frac{\alpha_{s} \alpha_{t}}{\alpha_{s t}}$ für jedes Elementenpaar $(s, t)$ von (3). Also ist die Zuordnung: $\quad U_{s} \rightarrow \sigma_{s}, A \rightarrow \chi(A)\left(A \in \mathfrak{F}_{s}(0)\right)$ eine ganze, nicht notwendig stetige, Darstellung 1-ten Grades von $\mathfrak{S}_{\mathfrak{S}}(0)$, daher ist $\chi$ ein Charakter von $\mathfrak{F}_{\mathfrak{S}}(0) / \mathfrak{F}^{\prime}$. Umgekehrt sei $\chi$ ein Charakter endlicher Ordnung von $\mathfrak{F}_{\mathscr{6}}(0) / \mathfrak{F}^{\prime}$, dann können wir $\chi$ zu einer ganzen, nicht notwendig stetigen, Darstellung von $\mathfrak{S}_{\mathbb{S}}(0) / \mathfrak{S}_{6}(0)^{\prime}$ erweitern : dies ist genau wie bei endlicher Gruppe zu zeigen, jedoch mit Hilfe der transfiniten Induktion.

Daraus ergibt sich

$$
m(\mathbb{S}) \cong \chi^{(e)}\left(\mathfrak{F}_{\mathscr{G}}(0)\right) / \chi^{(e)}\left(\mathfrak{F}_{\mathscr{G}}(0) / \mathfrak{\Im}^{\prime}\right),
$$

also gilt

$$
\mathfrak{M}\left((\mathfrak{S}) \cong \chi^{(e)}\left(\mathfrak{F}^{\prime}\right)\right.
$$

da (S) im Kleinen einfach-zusammenhängend ist, und folgiich $\mathfrak{F}_{\mathfrak{s}}(0)$ nach Satz 2 eine endliche Basis hat $\left.{ }^{14}\right)$.

(13) Vgl. J. von Neumann, Almost periodic function in a group, Trans. Amer. Math. Soc., 36 (1934), S. 482.

(14) sei eine abelsche Gruppe mit endlich vielen Erzeugenden, und $\mathfrak{2}$ eine Unter gruppe von ${ }_{0}$. Dann gilt wie üblich

$$
\chi(e)(\mathfrak{G}) / \chi(e)(\mathfrak{G} / \mathfrak{H}) \simeq \chi(e)(\mathfrak{F}) .
$$

Der Beweis folgt aus der Tatsache, dass jeder Charakter endlicher Ordnung von $\mathfrak{g} \mathrm{zu}$ einem Charakter endlicher Ordnung von $\mathcal{F}_{\text {e }}$ erweitert werden kann; und dies gilt unter der Voraussetzung, dass of endlich viele Erzeugende hat. Ohne diese Voraussetzung gilt das im allgemeinen nicht. 
Korollar. Der Multiplikator einer halbeinfachen kompakten Lieschen Gruppe ist mit der Fundamentatgruppe isomorph( $\left.{ }^{15}\right)$.

Denn die Fundamentalgruppe $\widetilde{F}_{\leftrightarrow}(0)$ ist endlich $\left({ }^{16}\right)$, und

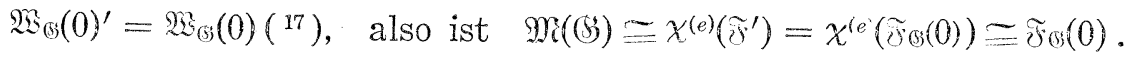

Es sei nun (5) kompakt metrisch, z.h. und im Kleinen z.h. aber nicht notwendig im Kleinen einfach-zusammenhängend. Dann ist $\widetilde{F}_{\mathfrak{c}}(0)$ die $\left(\mathfrak{S}_{n}\right.$-adische Limesgruppe der Folge $\left\{\mathcal{F}_{\mathfrak{S}}\left(n_{n}\right)\right\}$. Ist $\mathfrak{R}_{n}$ der Kern der stetigen

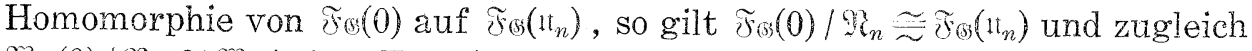
$\mathfrak{S}_{\mathfrak{G}}(0) / \mathfrak{M}_{n} \cong \mathfrak{S}_{\mathfrak{W}}\left(1 \mathrm{t}_{n}\right)$. Es sei noch $\mathfrak{R}_{n} / \mathfrak{N}_{n+1}=\mathfrak{B}_{n+1}$ und $\mathfrak{B}_{\mathfrak{s}}\left(\mathrm{tt}_{n}\right)^{\prime}$ die Kommutatorgruppe von $\mathfrak{S}_{\mathrm{s}}\left(1 \mathrm{tt}_{n}\right)$. Dann gilt

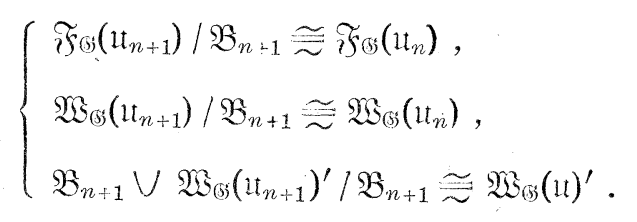

Die dritte Relation folgt daraus, dass $\mathfrak{B}_{n+1}$ im Zentrum von $\mathfrak{W}_{6 i}\left(\mathfrak{u}_{n+1}\right)$ enthalten ist. Setzt man $\widetilde{F}_{n}^{\prime}=\mathfrak{F}_{\mathfrak{F}}\left(\mathfrak{u t}_{n}\right) \cap \mathfrak{M}_{\mathfrak{G}}\left(\mathfrak{u}_{n}\right)^{\prime}$, so ist $\widetilde{F}_{n+1}^{\prime} / \widetilde{F}_{n+1}^{\prime} \cap \mathfrak{B}_{n+1} \stackrel{\approx}{\sim}$ $\mathfrak{B}_{n+1} \cup \mathfrak{F}_{n+1}^{\prime} / \mathfrak{B}_{n+1} \cong \widetilde{F}_{n}^{\prime}$, daher ist $\chi^{(e)}\left(\mathfrak{F}_{n}^{\prime}\right)$ als Untergruppe in $\chi^{(e)}\left(\widetilde{\digamma}_{n+1}^{\prime}\right)$ enthalten. Ganz analog wie (25) gilt

$$
\left\{\begin{array}{l}
\mathfrak{W}_{\omega}^{\prime}\left(\mathfrak{H}_{n}\right) \cong \mathfrak{W}_{\mathfrak{G}}(0)^{\prime} \cup \mathfrak{N}_{n} / \mathscr{Y}_{n} \\
\mathfrak{F}_{n}^{\prime} \cong \mathfrak{F}^{\prime} \cup \mathfrak{N}_{n} / \mathfrak{N}_{n} \cong \mathfrak{F}^{\prime} / \mathfrak{F}^{\prime} \cap \mathfrak{N}_{n} .
\end{array}\right.
$$

Also ist auch $\chi^{(e)}\left(\widetilde{\mho}_{n}^{\prime}\right)$ als Untergruppe in $\chi^{(e)}\left(\widetilde{\mho}^{\prime}\right)$ enthalten. Die Vereinigungsgruppe aller Untergruppen $\chi^{(e)}\left(\Re_{n}\right)(n=1,2, \ldots)$ von $\chi^{(e)}\left(\Re^{\prime}\right)$ sei mit

\section{bezeichnet. Dann gilt}

$$
\lim _{n} \chi^{(e)}\left(\widetilde{F}_{n}^{\prime}\right)
$$

Satz 15'. (5) sei eine kompakte, metrische zusammenhängende, im Kleinen zuasmmenhängende topologische Gruppe. Dann ist

(15) Für die eigentlich orthogonale Gruppe ist daher das zur Spindarstellung gehörige Faktorensystem das einzige, welches nicht mit 1 assoziiert ist, da nämlich die Fundamentalgruppe dieser Gruppe zyklisch von der Ordnung 2 ist. Aus Satz 14 ergibt sich also, dass alle S.P.D. der eigentlich orthogonalen Gruppe aus der S. G.D. und Spindarstellung konstruiert werden können. Vgl. dazu

H. Weyl, a.a.O. ( $\left.{ }^{3}\right)$, S. 270 , und 425-449.

R. Brauer and H. Weyl, Spinors in n-dimensions, Amer. Jour. of Math., 57 (1935),

(16) Vgl. H. Weyl, Theorie der Darstellung kontinuierlicher halbeinfacher Gruppen durch lineare Transformationen III, Math. Zeitschr., 24 (1926), S. 380, Satz 2.

(17) Vgl. B. L. van der Waerden, Stetigkeitssätze der halbeinfachen Lieschen Gruppen, Math. Zeitschr., 36 (1933), 780-786. 


$$
M\left((3) \cong \chi^{(e)}\left(\widetilde{F}_{(j)}(0)\right) / \chi^{(e)}\left(\widetilde{F}_{(j)}(0) / \widetilde{F}^{\prime}\right)\right.
$$

oder

$$
\operatorname{miG}\left(\subseteq \lim _{n} \chi^{(e)}\left(\widetilde{\Im}_{n}^{\prime}\right)<\chi^{(e)}\left(\widetilde{F}^{\prime}\right)\right.
$$

Beweis. (28) ist schon im Satz 15 bewiesen. Wie bei Satz 15 ist $\chi^{(e)}\left(\mathscr{\mho}_{n}^{\prime}\right)$ isomorph mit einer Untergruppe von $M((3)$. (29) folgt aus der Tatsache, dass ein beliebiges Element aus $M(\mathbb{S})$ in einer $\chi^{(e)}\left(\widetilde{\mho}_{n}^{\prime}\right)$ enthalten ist, wie im Satze 10 .gezeigt ist.

Als Anwendung der Sätze 15, 15' ergibt sich

Satz 16. (S) sei eine kompakte, metrische, zusammenhängende, und im Kleinen zusammenhängende abelsche Gruppe. Dann giti

$$
M(13)=1 \cdot\left({ }^{18}\right)
$$

Beweis. Da nach Satz $6 \mathfrak{W}_{6}(0)$ auch abelsch ist, ergibt sich $\widetilde{\mho}^{\prime}=1$, also $\mathfrak{M}(\mathbb{S}) \cong \lim \chi^{(e)}\left(\widetilde{\mho}_{n}^{\prime}\right)=1$.

Satz 17. Es seien (5) und (S) kompakte, metrische, zusammenhängende und im Kleinen zusammenhängende topologische Gruppen. Dann ist

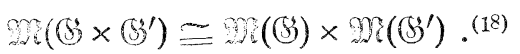

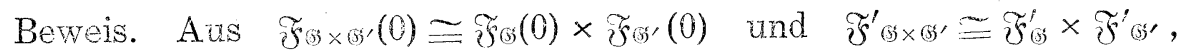
(nach Satz 8) folgt

$$
\begin{aligned}
& \chi^{(e)}\left(\widetilde{F}_{G \times G}(0)\right) \cong \chi^{(e)}\left(\widetilde{J}_{0}(0)\right) \times \chi^{(e)}\left(\widetilde{F}_{G}(0)\right)
\end{aligned}
$$

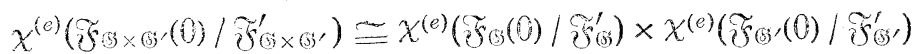

also nach Satz $15^{\prime}$

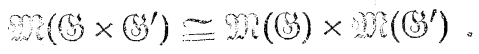

Zum Schluss beweisen wir einen Satz über die Homotopieeigenschaft der S.P.D.

Sarz 18. (5) und $\overline{2}_{1}$ seien zwei S.P.D. n-ten Grades einer kompatiten metrischen zusammenhängenden, im Kleinen zusammenhängenden Gruppe. Wenn $\bar{S}_{0}$ und $\mathbb{D}_{1}$ als stetige Abbildungen von $\$$ in $\mathfrak{P}_{n}$ homotop sind, dann sind $u_{a, b}^{(0)}$ und $u_{a, b}^{(1)}$ assoziiert, wobei $u_{a, b}^{(0)} b z w . u_{a, b}^{(\cdot 1)}$ das $z u \overline{2}_{0}$ bzw. ${ }_{1}$ gehörige Faktorensystem ist.

(18) Im Falle der endlichen Gruppe gilt $\mathfrak{M}=1$ für eine zykliche Gruppe, aber nicht im allgemeinen. Also gilt Satz 16 für endliche Gruppen nicht im allgemeinen. Vgl. dazu

R. Frucht, Über die Darstellung endlicher Abelscher Gruppen durch Kollineationen, Crelles Journal, 166 (1932), 16-29. 
Beweis. Aus der. Homotopie von $\overline{\mathfrak{D}}_{0}$ und $\overline{\mathfrak{D}}_{1}$ folgt die Homotopie von

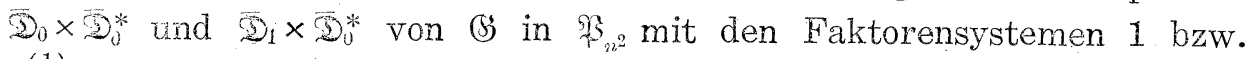
$u_{a, b}^{(1)} \cdot u_{a, b}^{(0)-1}$, wobei $5^{*}$ die konjugiert-komplexe Darstellung der unitären unimodularen Repräsentanten von $\overline{\mathfrak{D}}_{0}$ bedeutet. (Vgl. den Beweis von Satz 14.) Da $\overline{\mathfrak{D}}_{0} \times \overline{\mathfrak{D}}_{0}^{*}$ nach dem Korollar von Satz 13 für passend gewählte Repräsentanten $D(a) \in \bar{D}_{0}(a) \times D_{0}(a)^{*}$ eine G.S.D. wird, genügt es beim Beweis des Satzes nur den Fall zu zeigen, wo $\overline{\mathfrak{D}}_{0}$ die S.G.D. $a \rightarrow D(\alpha)$ ist. Denn daraus folgt, dass $u_{a, b}^{(1)} \cdot u_{p, b}^{(0)-1}$ mit 1 assoziiert ist, d.h. $u_{a, b}^{(0)}$ mit $u_{a, b}^{(0)}$ assoziiert ist.

Es sei $\bar{D}_{t}(0 \leqq t \leqq 1)$ eine Schar stetiger Abbildungen von $(3)$ in $\Re_{n}$, die als stetige Abbildungen von (5) in bezug auf $t$ stetig sind, wobei $\bar{D}_{0}$ und $\overline{\mathfrak{S}}_{1}$ mit der vorgegebenen übereinstimmen. Nun zeigen wir, dass ist.

$R(a, b)=\operatorname{Max}_{t \leq t \leq 1} \rho_{1}\left(\widehat{D_{t}(a)}, \widehat{D_{t}(b)}\right)$ eine stetige Funktion von $a, b \in(\mathfrak{s})$

Es sei $r(a, b)$ eine invariante Metrik von (3), die die vorgegebene Topologie induziert. Zu zeigen ist, dass für $r(a, b)<\delta(\varepsilon) \quad R(a, b)<\varepsilon$ ist. Wäre nün $R\left(a_{k}, b_{k}\right)>\varepsilon$ oder $\left.\rho_{1}\left({\overline{D_{t_{k}}\left(\alpha_{k}\right.}}\right), \widehat{D_{t_{k}}\left(b_{k}\right)}\right)>\varepsilon$ für $r\left(\alpha_{k}, b_{k}\right) \rightarrow 0$. Da (5) kompakt ist, können wir dabei o.B.d.A. annehmen, dass $a_{k} \rightarrow a_{0}, b_{k} \rightarrow b_{0}=a_{0}$ und $t_{k} \rightarrow t_{0}$. Wenn wir $\lambda$ so klein nehmen, dass $\rho_{1}\left(\widehat{D_{t}(a)}, \widehat{D_{t^{\prime}}(a)}\right)<\frac{\varepsilon}{3}$ für $\left|t-t^{\prime}\right|<\lambda$ ist, dann wäre für $\left|t,-t_{k}\right|<\lambda$

$$
\begin{aligned}
& \rho_{1}\left(\widetilde{D_{t_{0}}\left(a_{k}\right)}, \widetilde{D_{t_{0}}\left(b_{k}\right)}\right)>\rho_{1}\left(\widehat{D_{t_{k}}\left(a_{k}\right)}, \widehat{D_{t_{k}}\left(b_{k}\right)}\right)-\rho_{1}\left(\widetilde{D_{t_{k}}\left(a_{k}\right)}, \widetilde{D_{t_{0}}\left(a_{k}\right)}\right. \\
& \left.-\rho_{1}\left(\widetilde{D_{t_{k}}\left(b_{k}\right.}\right), \widehat{D_{t_{0}}\left(b_{k}\right)}\right)>\frac{\varepsilon}{3},
\end{aligned}
$$

was mit der Stetigkeit von $\overline{\text { S }}$ in Widerspruch steht.

Da $\mathfrak{P}_{n}$ und $\mathfrak{u}_{n}$ im Kleinen topologisch isomorph sind, können wir nun $D_{i}(a) \in U_{n}$ aus der Klasse $\widehat{D_{t}(a)} \in \Re_{n}(0 \leqq t \leqq 1)$ so wählen, dass $D_{t}(a)$ stetig von $t$ abhängt, und $D_{0}(a)$ mit der vorgegebenen S.G.D. $D_{0}(a)$ übereinstimmt. Dann zeigen wir, dass

$$
\rho_{0}\left(D_{t}(a), D_{t}(b)\right)=\rho_{1}\left(\widehat{D_{t}(a)}, \widehat{D_{t}(b)}\right) \quad(0 \leqq t \leqq 1)
$$

für $r(a, b)<\varepsilon$ mit genügend kleinen $\varepsilon>0$ gilt.

Für $t=0$ folgt (30) ans der Definition. Es gelte nun für $0 \leqq t \leqq t_{0}<1$. Dann ist 


$$
\begin{gathered}
\rho_{0}\left(D_{t}(a), D_{t}(b)\right) \leqq \rho_{1}\left(\widehat{D_{t_{0}}(a)}, \widetilde{D_{t_{0}}(b)}\right)+\rho_{0}\left(D_{t}(a), D_{t_{0}}(a)\right)+\rho_{0}\left(D_{t}(b), D_{t_{0}}(b)\right) \\
\leqq \rho_{0}\left(D_{t}(a), D_{t}(b) \omega_{n}^{i}\right)
\end{gathered}
$$

für $\left|t-t_{0}\right|<\lambda$ mit genügend kleinen $\lambda>0$, da

$$
\begin{aligned}
& \rho_{1}\left(\widetilde{D_{t_{0}}(a)}, \widetilde{D_{t_{0}}(b)}\right) \leqq R(a, b)<\left|1-\omega_{n}^{i}\right| \sqrt{n}-R(a, b) \\
\leqq & \rho_{0}\left(D_{t_{0}}(b), D_{t_{0}}(b) \omega_{n}^{i}\right)-\rho_{0}\left(D_{t_{0}}(a), D_{t_{0}}(b)\right) \\
\leqq & \rho_{0}\left(D_{t_{0}}(a), D_{t_{0}}(b) \omega_{n}^{i}\right) \quad(i \neq 0(n))
\end{aligned}
$$

für $r(a, b)<\varepsilon$ ist. Also gilt (30) für $0 \leqq t \leqq t_{0}+\lambda$, also schliesslich für $0 \leqq t \leqq 1$, da (30) auf eine abgeschlossene Menge von $t$ von [1,0] gilt. Es gilt daher für $r(a, b)<\varepsilon$

$$
\rho_{0}\left(D_{1}(a), D_{1}(b)\right)=\rho_{1}\left(\widehat{D_{1}(a)}, \widehat{D_{1}(b)}\right) \leqq R(a, b) .
$$

Darus ergibt sich, dass $D_{1}(a) \in \mathfrak{U}_{n}$ eine stetige Funktion von $a \in \mathbb{G}$ ist. Da aber (5) z.h. ist, und $u_{a, b}^{(1)}=D(a) \cdot D(b) \cdot D(a b)^{-1}$ stetig von $a, b$ abhängt, folgt $u_{a, b}^{(1)}=1$ aus $u_{a, b}^{(1) n}=1$.

Mathematisches Institut,

Kaiserliche Universität zu Tokyo. 